Supporting Information for

\title{
Visible-Light-Promoted Dual C-C Bond Formations of alkynoates via a domino radical addition/cyclization reaction: A Synthesis of Coumarins
}

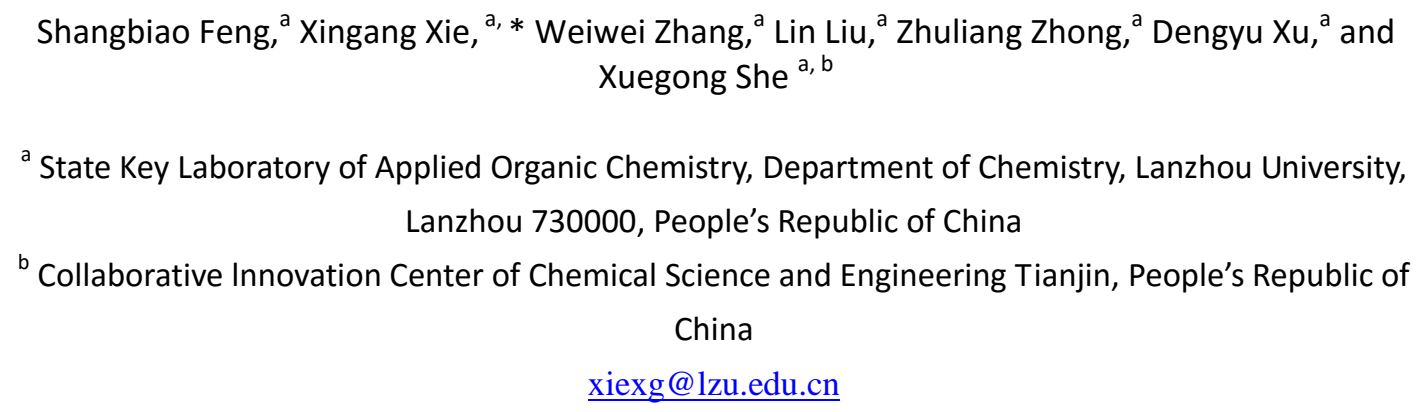

\section{TABLE OF CONTENTS}

1. General information

2. Preparation of starting materials

3. General procedure for the domino radical addition/cyclization reaction

4. Characterization data for all products

5. Kinetic isotope effect experiment

6. ${ }^{1} \mathrm{H}$ and ${ }^{13} \mathrm{C}$ NMR spectra of the products 


\section{General information.}

Solvents were purified and dried by standard methods prior to use. All commercially available reagents were used without further purification unless otherwise noted. All syntheses of complex were carried out under argon atmosphere on Wattecs Parallel Reactor. Column chromatography was generally performed on silica gel (200-300 mesh) and reactions were monitored by thin layer chromatography (TLC) using silica gel GF254 plates with UV light to visualize the course of reaction. Melting points were determined with a digital Koffer apparatus and were uncorrected. ${ }^{1} \mathrm{H}$ and ${ }^{13} \mathrm{C}$ NMR data were recorded on a 400 $\mathrm{MHz}$ spectrometer using $\mathrm{CDCl}_{3}$ as solvent at room temperature. The chemical shifts $(\delta)$ are reported in ppm and coupling constants $(J)$ in Hz. High-resolution mass spectra (HRMS) were obtained on a FT-ICR spectrometer.

\section{Preparation of starting materials. ${ }^{[1]}$}

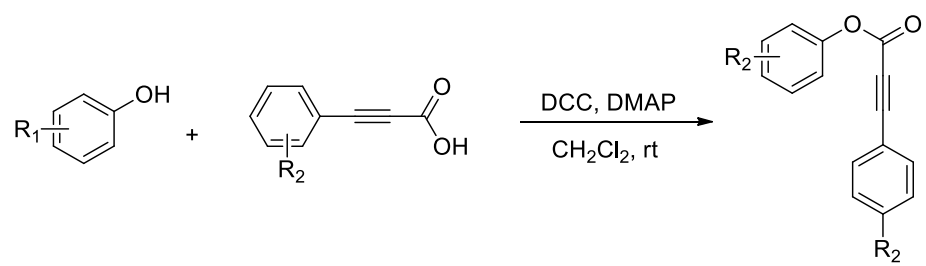

To a solution of the phenol $\left(5.0 \mathrm{mmol}, 1.0\right.$ equiv) in $\mathrm{CH}_{2} \mathrm{Cl}_{2}(20 \mathrm{~mL})$ was added 3-phenylpropiolic acid (5.5 mmol, 1.1 equiv) at $0{ }^{\circ} \mathrm{C}$, then a mixture of DCC $(7.5 \mathrm{mmol}, 1.5$ equiv) and DMAP ( $0.5 \mathrm{mmol}, 0.1$ equiv) in $\mathrm{CH}_{2} \mathrm{Cl}_{2}(10 \mathrm{~mL})$ was added dropwise. The resulting mixture was stirred at room temperature for 12 hours. Then the crude mixture was filtered and washed with $\mathrm{CH}_{2} \mathrm{Cl}_{2}(20 \mathrm{~mL})$. The combined organic phase was concentrated under reduced pressure to give a residue which was purified by a silica gel column chromatography (petroleum ether/EtOAc $=7: 1$ ) to give the alkynoate products.

\section{General procedure for the domino radical addition/cyclization reaction.}

To a reaction tube equipped with a magnetic stir bar was added phenyl 3-phenylpropiolate ( $0.15 \mathrm{mmol}, 1.0$ equiv), $\mathrm{Ru}(\mathrm{bpy})_{3} \mathrm{Cl}_{2} \cdot 6 \mathrm{H}_{2} \mathrm{O}(5 \mathrm{~mol} \%, 6 \mathrm{mg})$ in mixed solvent of $\mathrm{MeCN}(1.0 \mathrm{~mL})$ and THF (3.0 mL) was added $t-\mathrm{BuOOH}(5.5 \mathrm{M}$ in decane) $(0.6 \mathrm{mmol}, 4.0$ equiv). Then the reaction was irradiated with a $34 \mathrm{~W}$ blue LED strip and stirred at ambient temperature from 40-48 hours. The reaction was monitored by TLC to establish the consumption of starting material. After it was complete, the reaction mixture was concentrated under 
reduced pressure to give a residue which was purified by silica gel column chromatography to afford the desired coumarin product.

\section{Characterization data for all products.}

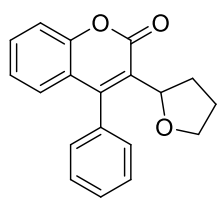

$3 a$

Compound 3a was obtained as a white solid in $64 \%$ yield according to the general procedure. Mp: $179-181^{\circ} \mathrm{C} .{ }^{1} \mathrm{H}^{\mathrm{NMR}}\left(400 \mathrm{MHz}, \mathrm{CDCl}_{3}\right) \delta 7.58-7.43(\mathrm{~m}, 4 \mathrm{H}), 7.40-7.29(\mathrm{~m}, 2 \mathrm{H}), 7.23-$ $7.16(\mathrm{~m}, 1 \mathrm{H}), 7.15-7.07(\mathrm{~m}, 1 \mathrm{H}), 6.95(\mathrm{dd}, J=8.0,1.3 \mathrm{~Hz}, 1 \mathrm{H}), 4.68-4.61(\mathrm{~m}, 1 \mathrm{H}), 4.04(\mathrm{td}, J$ $=8.1,6.4 \mathrm{~Hz}, 1 \mathrm{H}), 3.78(\mathrm{td}, J=7.6,3.9 \mathrm{~Hz}, 1 \mathrm{H}), 2.37(\mathrm{dq}, J=11.6,8.7 \mathrm{~Hz}, 1 \mathrm{H}), 2.22-2.08(\mathrm{~m}$, 1H), $2.04-1.89(\mathrm{~m}, 1 \mathrm{H}), 1.84(\mathrm{ddt}, J=16.9,11.7,8.3 \mathrm{~Hz}, 1 \mathrm{H}) .{ }^{13} \mathrm{C} \mathrm{NMR}\left(100 \mathrm{MHz}, \mathrm{CDCl}_{3}\right) \delta$ $159.4,153.0,152.4,134.2,131.3,128.8,128.7,128.5,128.2,127.7,125.8,123.9,120.5$, 116.5, 76.5, 69.5, 30.2, 27.4. HRMS (ESI): $\mathrm{m} / \mathrm{z}[\mathrm{M}+\mathrm{H}]^{+}$calculated for $\mathrm{C}_{19} \mathrm{H}_{17} \mathrm{O}_{3}: 293.1172$, found: 293.1170 .

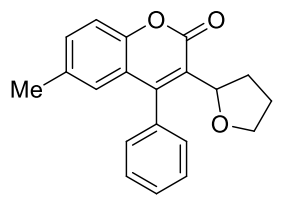

3b

Compound $\mathbf{3 b}$ was obtained as a white solid in $72 \%$ yield according to the general procedure. Mp: $136-138^{\circ} \mathrm{C} .{ }^{1} \mathrm{H}_{\text {NMR }}\left(400 \mathrm{MHz}, \mathrm{CDCl}_{3}\right) \delta 7.56-7.44(\mathrm{~m}, 3 \mathrm{H}), 7.34-7.29(\mathrm{~m}, 1 \mathrm{H}), 7.21-$ $7.13(\mathrm{~m}, 2 \mathrm{H}), 6.92(\mathrm{dd}, J=8.2,1.0 \mathrm{~Hz}, 1 \mathrm{H}), 6.82(\mathrm{~d}, J=8.1 \mathrm{~Hz}, 1 \mathrm{H}), 4.68-4.57(\mathrm{~m}, 1 \mathrm{H}), 4.04$ $(\mathrm{td}, J=8.1,6.3 \mathrm{~Hz}, 1 \mathrm{H}), 3.77(\mathrm{td}, J=7.6,4.0 \mathrm{~Hz}, 1 \mathrm{H}), 2.42(\mathrm{~s}, 3 \mathrm{H}), 2.35$ (ddd, $J=11.5,8.8,3.8$ $\mathrm{Hz}, 1 \mathrm{H}), 2.20-2.09(\mathrm{~m}, 1 \mathrm{H}), 1.99-1.90(\mathrm{~m}, 1 \mathrm{H}), 1.89-1.77(\mathrm{~m}, 1 \mathrm{H}) .{ }^{13} \mathrm{C}$ NMR $(100 \mathrm{MHz}$ $\left.\mathrm{CDCl}_{3}\right) \delta 159.6,153.1,152.5,142.4,134.4,128.7,128.6,128.6,128.4,128.2,127.4,125.1$, 124.6, 118.1, 116.6, 76.5, 69.5, 30.1, 27.4, 21.5. HRMS (ESI): $\mathrm{m} / \mathrm{z}[\mathrm{M}+\mathrm{H}]^{+}$calculated for $\mathrm{C}_{20} \mathrm{H}_{19} \mathrm{O}_{3}: 307.1329$, found: 307.1333 .<smiles>CCc1ccc2oc(=O)c(C3CCCO3)c(-c3ccccc3)c2c1</smiles>

Compound $3 \mathbf{c}$ was obtained as ropy oil in $71 \%$ yield according to the general procedure. ${ }^{1} \mathbf{H}$ 
NMR $\left(400 \mathrm{MHz}, \mathrm{CDCl}_{3}\right) \delta 7.54-7.45(\mathrm{~m}, 3 \mathrm{H}), 7.33(\mathrm{~d}, J=1.4 \mathrm{~Hz}, 1 \mathrm{H}), 7.18(\mathrm{dd}, J=4.1,2.4 \mathrm{~Hz}$, 2H), $6.95(\mathrm{dd}, J=8.2,1.6 \mathrm{~Hz}, 1 \mathrm{H}), 6.85(\mathrm{~d}, J=8.2 \mathrm{~Hz}, 1 \mathrm{H}), 4.66-4.59(\mathrm{~m}, 1 \mathrm{H}), 4.04(\mathrm{td}, J=8.1$, $6.3 \mathrm{~Hz}, 1 \mathrm{H}), 3.77(\mathrm{td}, J=7.7,4.0 \mathrm{~Hz}, 1 \mathrm{H}), 2.71(\mathrm{q}, J=7.6 \mathrm{~Hz}, 2 \mathrm{H}), 2.36(\mathrm{dq}, J=11.6,8.7 \mathrm{~Hz}, 1 \mathrm{H})$ $2.19-2.09(\mathrm{~m}, 1 \mathrm{H}), 1.99-1.90(\mathrm{~m}, 1 \mathrm{H}), 1.89-1.76(\mathrm{~m}, 1 \mathrm{H}), 1.25(\mathrm{t}, J=7.6 \mathrm{~Hz}, 3 \mathrm{H}) .{ }^{13} \mathrm{C}$ NMR $\left(100 \mathrm{MHz}, \mathrm{CDCl}_{3}\right) \delta 159.6,153.2,152.5,148.7,134.4,128.7,128.6,128.6,128.4,128.2,127.5$, 124.6, 123.9, 118.3, 115.4, 76.5, 69.5, 30.2, 28.7, 27.4, 15.1. HRMS (ESI): $m / z[M+H]^{+}$ calculated for $\mathrm{C}_{21} \mathrm{H}_{21} \mathrm{O}_{3}: 321.1485$, found: 321.1484 .

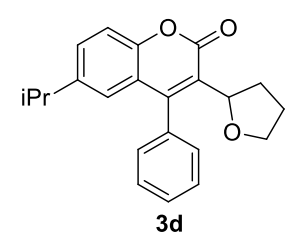

Compound $\mathbf{3 d}$ was obtained as a white solid in $82 \%$ yield according to the general procedure. Mp: $99-100^{\circ} \mathrm{C} .{ }^{1} \mathrm{H}$ NMR $\left(400 \mathrm{MHz}, \mathrm{CDCl}_{3}\right) \delta 7.54-7.45(\mathrm{~m}, 3 \mathrm{H}), 7.35-7.30(\mathrm{~m}, 1 \mathrm{H}), 7.21(\mathrm{~d}, \mathrm{~J}$ $=1.6 \mathrm{~Hz}, 1 \mathrm{H}), 7.18(\mathrm{dt}, J=6.2,2.0 \mathrm{~Hz}, 1 \mathrm{H}), 6.99(\mathrm{dd}, J=8.3,1.5 \mathrm{~Hz}, 1 \mathrm{H}), 6.86(\mathrm{~d}, J=8.2 \mathrm{~Hz}$, 1H), $4.67-4.60(\mathrm{~m}, 1 \mathrm{H}), 4.04(\mathrm{td}, J=8.1,6.3 \mathrm{~Hz}, 1 \mathrm{H}), 3.77(\mathrm{td}, J=7.6,4.0 \mathrm{~Hz}, 1 \mathrm{H}), 2.96(\mathrm{dt}, J$ $=13.8,6.9 \mathrm{~Hz}, 1 \mathrm{H}), 2.35(\mathrm{dq}, J=11.5,8.7 \mathrm{~Hz}, 1 \mathrm{H}), 2.19-2.08(\mathrm{~m}, 1 \mathrm{H}), 1.98-1.89(\mathrm{~m}, 1 \mathrm{H})$, $1.89-1.76(\mathrm{~m}, 1 \mathrm{H}), 1.26(\mathrm{~d}, J=6.9 \mathrm{~Hz}, 6 \mathrm{H}) .{ }^{13} \mathrm{C}$ NMR $\left(100 \mathrm{MHz}, \mathrm{CDCl}_{3}\right) \delta 159.6,153.4,153.2$, $152.4,134.5,128.7,128.6,128.6,128.4,128.2,127.6,124.7,122.6,118.4,114.0,76.5,69.5$, 34.1, 30.2, 27.4, 23.6. HRMS (ESI): $\mathrm{m} / \mathrm{z}[\mathrm{M}+\mathrm{H}]^{+}$calculated for $\mathrm{C}_{22} \mathrm{H}_{23} \mathrm{O}_{3}: 335.1642$, found: 335.1648.

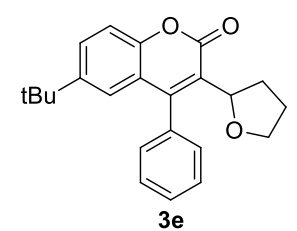

Compound 3e was obtained as a white solid in $82 \%$ yield according to the general procedure. Mp: $104-106^{\circ} \mathrm{C} .{ }^{1} \mathrm{H}$ NMR $\left(400 \mathrm{MHz}, \mathrm{CDCl}_{3}\right) \delta 7.54-7.45$ (m, 3H), $7.36(\mathrm{~d}, J=1.8 \mathrm{~Hz}, 1 \mathrm{H}), 7.34$ $-7.30(\mathrm{~m}, 1 \mathrm{H}), 7.21-7.13(\mathrm{~m}, 2 \mathrm{H}), 6.88(\mathrm{~d}, J=8.4 \mathrm{~Hz}, 1 \mathrm{H}), 4.70-4.59(\mathrm{~m}, 1 \mathrm{H}), 4.04(\mathrm{td}, J=$ 8.1, 6.4 Hz, 1H), $3.77(\mathrm{td}, J=7.6,4.0 \mathrm{~Hz}, 1 \mathrm{H}), 2.35(\mathrm{dq}, J=11.5,8.7 \mathrm{~Hz}, 1 \mathrm{H}), 2.21-2.08(\mathrm{~m}$, 1H), $2.01-1.90(\mathrm{~m}, 1 \mathrm{H}), 1.83$ (ddt, $J=16.8,11.7,8.2 \mathrm{~Hz}, 1 \mathrm{H}), 1.33(\mathrm{~s}, 9 \mathrm{H}) .{ }^{13} \mathrm{C}$ NMR (100 MHz, $\left.\mathrm{CDCl}_{3}\right) \delta 159.7,155.7,153.0,152.3,134.4,128.7,128.6,128.6,128.4,128.2,127.2,124.8$, 121.4, 118.0, 113.3, 76.5, 69.5, 35.1, 31.0, 30.2, 27.4. HRMS (ESI): $m / z[M+H]^{+}$calculated for 
$\mathrm{C}_{23} \mathrm{H}_{25} \mathrm{O}_{3}: 349.1798$, found: 349.1800 .

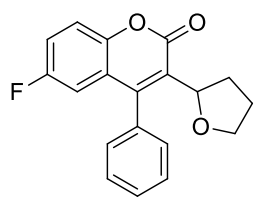

$3 \mathrm{~g}$

Compound $3 \mathrm{~g}$ was obtained as a white solid in $54 \%$ yield according to the general procedure.

Mp: $174-176^{\circ} \mathrm{C} .{ }^{1} \mathrm{H}$ NMR $\left(400 \mathrm{MHz}, \mathrm{CDCl}_{3}\right) \delta 7.56-7.47(\mathrm{~m}, 3 \mathrm{H}), 7.33-7.29(\mathrm{~m}, 1 \mathrm{H}), 7.18(\mathrm{dt}$, $J=6.1,2.1 \mathrm{~Hz}, 1 \mathrm{H}), 7.07(\mathrm{dd}, J=8.9,2.5 \mathrm{~Hz}, 1 \mathrm{H}), 6.94(\mathrm{dd}, J=8.9,6.1 \mathrm{~Hz}, 1 \mathrm{H}), 6.85(\mathrm{td}, J=8.5$, $2.5 \mathrm{~Hz}, 1 \mathrm{H}), 4.65-4.58(\mathrm{~m}, 1 \mathrm{H}), 4.03(\mathrm{td}, J=8.1,6.3 \mathrm{~Hz}, 1 \mathrm{H}), 3.78(\mathrm{td}, J=7.7,4.0 \mathrm{~Hz}, 1 \mathrm{H})$, $2.35(\mathrm{ddd}, J=17.5,11.7,8.7 \mathrm{~Hz}, 1 \mathrm{H}), 2.19-2.09(\mathrm{~m}, 1 \mathrm{H}), 2.00-1.91(\mathrm{~m}, 1 \mathrm{H}), 1.83(\mathrm{ddt}, J=$ 16.8, 11.7, 8.2 Hz, 1H). ${ }^{13} \mathrm{C}$ NMR $\left(100 \mathrm{MHz}, \mathrm{CDCl}_{3}\right) \delta(165.32,162.80, \mathrm{~d}, J=252 \mathrm{~Hz}), 158.99$, (154.11, 153.99, d, J = $12 \mathrm{~Hz}), 151.95,134.02,129.54,129.44,128.91,128.85,128.58,128.15$, (124.69, 124.66, d, $J=3 \mathrm{~Hz}),(117.30,117.28, d, J=2 \mathrm{~Hz}),(112.02,111.80, d, J=22 \mathrm{~Hz})$, $(104.01,103.75, \mathrm{~d}, J=26 \mathrm{~Hz}), 76.40,69.54,30.14,27.39 .{ }^{19} \mathrm{~F}$ NMR $\left(376 \mathrm{MHz}, \mathrm{CDCl}_{3}\right) \delta-106.37$. HRMS (ESI): $m / z[\mathrm{M}+\mathrm{H}]^{+}$calculated for $\mathrm{C}_{19} \mathrm{H}_{16} \mathrm{FO}_{3}: 311.1078$, found: 311.1083 .<smiles>O=c1oc2ccc(Cl)cc2c(-c2ccccc2)c1C1CCCO1</smiles>

Compound $3 \mathrm{~h}$ was obtained as a white solid in $51 \%$ yield according to the general procedure. Mp: $167-169^{\circ} \mathrm{C} .{ }^{1} \mathrm{H}$ NMR $\left(400 \mathrm{MHz}, \mathrm{CDCl}_{3}\right) \delta 7.59-7.45(\mathrm{~m}, 3 \mathrm{H}), 7.36(\mathrm{~d}, J=2.0 \mathrm{~Hz}, 1 \mathrm{H}), 7.33$ $-7.28(\mathrm{~m}, 1 \mathrm{H}), 7.18(\mathrm{dt}, J=6.1,2.1 \mathrm{~Hz}, 1 \mathrm{H}), 7.08(\mathrm{dd}, J=8.6,2.0 \mathrm{~Hz}, 1 \mathrm{H}), 6.88(\mathrm{~d}, J=8.6 \mathrm{~Hz}$, $1 \mathrm{H}), 4.67-4.57(\mathrm{~m}, 1 \mathrm{H}), 4.02(\mathrm{td}, J=8.2,6.4 \mathrm{~Hz}, 1 \mathrm{H}), 3.78(\mathrm{td}, J=7.7,3.9 \mathrm{~Hz}, 1 \mathrm{H}), 2.34(\mathrm{dq}, J$ $=11.6,8.7 \mathrm{~Hz}, 1 \mathrm{H}), 2.22-2.08(\mathrm{~m}, 1 \mathrm{H}), 2.03-1.90(\mathrm{~m}, 1 \mathrm{H}), 1.84(\mathrm{ddt}, J=16.9,11.8,8.3 \mathrm{~Hz}$, 1H). ${ }^{13} \mathrm{C}$ NMR $\left(100 \mathrm{MHz}, \mathrm{CDCl}_{3}\right) \delta 158.7,153.2,151.7,137.2,133.8,128.9,128.9,128.7,128.6$, 128.6, 128.2, 125.9, 124.5, 119.2, 116.7, 76.4, 69.6, 30.2, 27.4. HRMS (ESI): $m / z[M+H]^{+}$ calculated for $\mathrm{C}_{19} \mathrm{H}_{16} \mathrm{ClO}_{3}: 327.0782$, found: 327.0781 .<smiles>O=c1oc2ccc(Br)cc2c(-c2ccccc2)c1C1CCCO1</smiles>

3i

Compound $3 \mathbf{i}$ was obtained as a white solid in $56 \%$ yield according to the general procedure. 
Mp: $150-152^{\circ} \mathrm{C}^{1}{ }^{\mathrm{H}} \mathrm{NMR}\left(400 \mathrm{MHz}, \mathrm{CDCl}_{3}\right) \delta 7.59-7.44(\mathrm{~m}, 4 \mathrm{H}), 7.30(\mathrm{dd}, J=6.5,1.5 \mathrm{~Hz}, 1 \mathrm{H})$, $7.23(\mathrm{dd}, J=8.6,1.8 \mathrm{~Hz}, 1 \mathrm{H}), 7.21-7.15(\mathrm{~m}, 1 \mathrm{H}), 6.81(\mathrm{~d}, J=8.6 \mathrm{~Hz}, 1 \mathrm{H}), 4.62(\mathrm{t}, J=8.1 \mathrm{~Hz}$, 1H), $4.08-3.95(\mathrm{~m}, 1 \mathrm{H}), 3.78(\mathrm{td}, J=7.7,3.9 \mathrm{~Hz}, 1 \mathrm{H}), 2.34(\mathrm{dq}, J=11.5,8.7 \mathrm{~Hz}, 1 \mathrm{H}), 2.23-$ $2.07(\mathrm{~m}, 1 \mathrm{H}), 2.01-1.91(\mathrm{~m}, 1 \mathrm{H}), 1.90-1.77(\mathrm{~m}, 1 \mathrm{H}) .{ }^{13} \mathrm{C} \mathrm{NMR}\left(100 \mathrm{MHz}, \mathrm{CDCl}_{3}\right) \delta 158.6$, $153.1,151.8,133.7,128.9,128.9,128.8,128.6,128.6,128.1,127.3,126.1,125.2,119.6$, 119.6, 76.4, 69.6, 30.2, 27.4. HRMS (ESI): $\mathrm{m} / \mathrm{z}[\mathrm{M}+\mathrm{H}]^{+}$calculated for $\mathrm{C}_{19} \mathrm{H}_{16} \mathrm{BrO}_{3}: 371.0277$, found: 371.0281 .

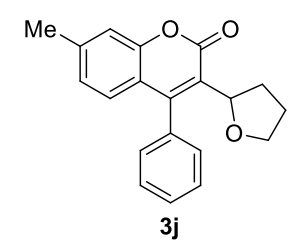

Compound $\mathbf{3} \mathbf{j}$ was obtained as a white solid in $46 \%$ yield according to the general procedure. Mp: $50-52^{\circ} \mathrm{C} .{ }^{1} \mathrm{H}$ NMR $\left(400 \mathrm{MHz}, \mathrm{CDCl}_{3}\right) \delta 7.56-7.46(\mathrm{~m}, 3 \mathrm{H}), 7.32(\mathrm{dd}, J=6.6,1.6 \mathrm{~Hz}, 1 \mathrm{H})$, $7.29-7.23(\mathrm{~m}, 2 \mathrm{H}), 7.21-7.16(\mathrm{~m}, 1 \mathrm{H}), 6.70(\mathrm{~s}, 1 \mathrm{H}), 4.65-4.57(\mathrm{~m}, 1 \mathrm{H}), 4.04(\mathrm{td}, J=8.0,6.3$ $\mathrm{Hz}, 1 \mathrm{H}), 3.77(\mathrm{td}, J=7.6,3.8 \mathrm{~Hz}, 1 \mathrm{H}), 2.36(\mathrm{ddd}, J=17.6,11.5,8.7 \mathrm{~Hz}, 1 \mathrm{H}), 2.24(\mathrm{~s}, 3 \mathrm{H}), 2.20-$ $2.09(\mathrm{~m}, 1 \mathrm{H}), 1.99-1.90(\mathrm{~m}, 1 \mathrm{H}), 1.83(\mathrm{ddt}, J=16.8,11.7,8.4 \mathrm{~Hz}, 1 \mathrm{H}) .{ }^{13} \mathrm{C}$ NMR $(100 \mathrm{MHz}$, $\left.\mathrm{CDCl}_{3}\right) \delta 159.5,152.4,151.2,134.4,133.5,132.3,128.8,128.7,128.6,128.4,128.2,127.4$, 125.7, 120.2, 116.2, 76.6, 69.5, 30.2, 27.4, 20.9. HRMS (ESI): $\mathrm{m} / \mathrm{z}[\mathrm{M}+\mathrm{H}]^{+}$calculated for $\mathrm{C}_{20} \mathrm{H}_{19} \mathrm{O}_{3}$ : 307.1329, found: 307.1328 .

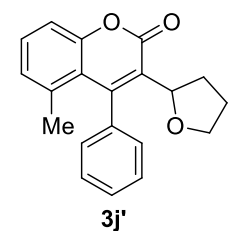

Compound $\mathbf{3} \mathbf{j}^{\prime}$ was obtained as a white solid in $31 \%$ yield according to the general procedure. Mp: $45-46^{\circ} \mathrm{C} .{ }^{1} \mathrm{H}$ NMR $\left(400 \mathrm{MHz}, \mathrm{CDCl}_{3}\right) \delta 7.54-7.45(\mathrm{~m}, 3 \mathrm{H}), 7.32$ (ddd, J = 6.8, 4.4, $1.3 \mathrm{~Hz}$, 2H), $7.17(\mathrm{~d}, J=1.7 \mathrm{~Hz}, 1 \mathrm{H}), 7.01(\mathrm{t}, J=7.7 \mathrm{~Hz}, 1 \mathrm{H}), 6.80-6.75(\mathrm{~m}, 1 \mathrm{H}), 4.68-4.60(\mathrm{~m}, 1 \mathrm{H})$, $4.04(\mathrm{td}, J=8.2,6.3 \mathrm{~Hz}, 1 \mathrm{H}), 3.78(\mathrm{td}, J=7.6,3.9 \mathrm{~Hz}, 1 \mathrm{H}), 2.49$ (s, 3H), 2.37 (ddd, $J=17.7,11.7$, $8.7 \mathrm{~Hz}, 1 \mathrm{H}), 2.20-2.10(\mathrm{~m}, 1 \mathrm{H}), 2.00-1.91(\mathrm{~m}, 1 \mathrm{H}), 1.90-1.77(\mathrm{~m}, 1 \mathrm{H}) .{ }^{13} \mathrm{C} \mathrm{NMR}(100 \mathrm{MHz}$, $\left.\mathrm{CDCl}_{3}\right) \delta 159.5,152.7,151.3,134.6,132.5,128.7,128.5,128.4,128.3,125.8,125.5,123.3$, 120.3, 76.6, 69.6, 30.2, 27.5, 15.6. HRMS (ESI): $\mathrm{m} / z[\mathrm{M}+\mathrm{H}]^{+}$calculated for $\mathrm{C}_{20} \mathrm{H}_{19} \mathrm{O}_{3}: 307.1329$, found: 307.1330 . 


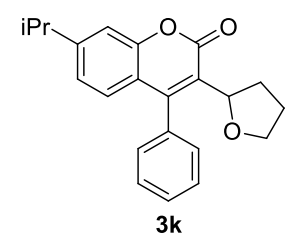

Compound $3 \mathbf{k}$ was obtained as a white solid in $60 \%$ yield according to the general procedure. Mp: $117-119^{\circ} \mathrm{C} .{ }^{1} \mathrm{H}$ NMR $\left(400 \mathrm{MHz}, \mathrm{CDCl}_{3}\right) \delta 7.57-7.47(\mathrm{~m}, 3 \mathrm{H}), 7.34$ (ddd, $J=10.0,7.7,1.9$ $\mathrm{Hz}, 2 \mathrm{H}), 7.29(\mathrm{~d}, J=8.5 \mathrm{~Hz}, 1 \mathrm{H}), 7.23-7.17(\mathrm{~m}, 1 \mathrm{H}), 6.74(\mathrm{~d}, J=2.0 \mathrm{~Hz}, 1 \mathrm{H}), 4.67-4.58(\mathrm{~m}$, $1 \mathrm{H}), 4.04(\mathrm{td}, J=8.1,6.4 \mathrm{~Hz}, 1 \mathrm{H}), 3.78(\mathrm{td}, J=7.6,3.9 \mathrm{~Hz}, 1 \mathrm{H}), 2.79(\mathrm{dt}, J=13.8,6.9 \mathrm{~Hz}, 1 \mathrm{H})$, $2.36(\mathrm{dq}, J=11.5,8.7 \mathrm{~Hz}, 1 \mathrm{H}), 2.20-2.07(\mathrm{~m}, 1 \mathrm{H}), 2.00-1.90(\mathrm{~m}, 1 \mathrm{H}), 1.83(\mathrm{ddt}, J=16.9$, 11.7, 8.3 Hz, 1H), 1.12 (dd, $J=6.9,1.6 \mathrm{~Hz}, 6 \mathrm{H}) .{ }^{13} \mathrm{C}$ NMR $\left(100 \mathrm{MHz}, \mathrm{CDCl}_{3}\right) \delta 159.6,152.5$, $151.3,144.6,134.3,129.5,128.7,128.7,128.6,128.4,128.3,125.6,125.1,120.2,116.4,76.6$, 69.5, 33.6, 30.2, 27.4, 23.9. HRMS (ESI): $\mathrm{m} / 2[\mathrm{M}+\mathrm{H}]^{+}$calculated for $\mathrm{C}_{22} \mathrm{H}_{23} \mathrm{O}_{3}: 335.1642$, found: 335.1640

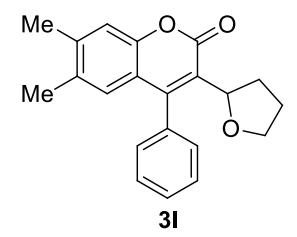

Compound $\mathbf{3 I}$ was obtained as a white solid in $42 \%$ yield according to the general procedure. Mp: $123-125^{\circ} \mathrm{C} .{ }^{1} \mathrm{H}$ NMR $\left(400 \mathrm{MHz}, \mathrm{CDCl}_{3}\right) \delta 7.55-7.45(\mathrm{~m}, 3 \mathrm{H}), 7.35-7.29(\mathrm{~m}, 1 \mathrm{H}), 7.17(\mathrm{dt}$ $J=6.2,2.0 \mathrm{~Hz}, 1 \mathrm{H}), 7.13(\mathrm{~s}, 1 \mathrm{H}), 6.65(\mathrm{~s}, 1 \mathrm{H}), 4.65-4.56(\mathrm{~m}, 1 \mathrm{H}), 4.04(\mathrm{td}, J=8.1,6.3 \mathrm{~Hz}, 1 \mathrm{H})$, $3.77(\mathrm{td}, J=7.6,3.9 \mathrm{~Hz}, 1 \mathrm{H}), 2.37(\mathrm{ddd}, J=11.6,8.7,4.0 \mathrm{~Hz}, 1 \mathrm{H}), 2.32(\mathrm{~s}, 3 \mathrm{H}), 2.19-2.09(\mathrm{~m}$, 4H), $1.98-1.89(\mathrm{~m}, 1 \mathrm{H}), 1.82(\mathrm{ddt}, J=16.9,11.8,8.2 \mathrm{~Hz}, 1 \mathrm{H}) .{ }^{13} \mathrm{C} \mathrm{NMR}\left(100 \mathrm{MHz}, \mathrm{CDCl}_{3}\right) \delta$ $159.8,152.5,151.4,141.3,134.5,132.6,128.7,128.6,128.5,128.4,128.3,127.7,124.5$, 118.2, 117.0, 76.6, 69.5, 30.1, 27.4, 20.1, 19.3. HRMS (ESI): $\mathrm{m} / \mathrm{z}[\mathrm{M}+\mathrm{H}]^{+}$calculated for $\mathrm{C}_{21} \mathrm{H}_{21} \mathrm{O}_{3}: 321.1485$, found: 321.1483 .

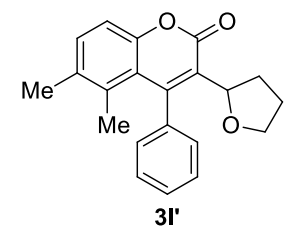

Compound $\mathbf{3 I}$ was obtained as ropy oil in $31 \%$ yield according to the general procedure. ${ }^{1} \mathbf{H}$ NMR $\left(400 \mathrm{MHz}, \mathrm{CDCl}_{3}\right) \delta 7.55-7.44(\mathrm{~m}, 3 \mathrm{H}), 7.30(\mathrm{~d}, J=6.5 \mathrm{~Hz}, 1 \mathrm{H}), 7.17(\mathrm{~d}, J=6.7 \mathrm{~Hz}, 1 \mathrm{H})$, $6.91(\mathrm{~d}, J=8.1 \mathrm{~Hz}, 1 \mathrm{H}), 6.67(\mathrm{~d}, J=8.1 \mathrm{~Hz}, 1 \mathrm{H}), 4.63(\mathrm{t}, J=8.0 \mathrm{~Hz}, 1 \mathrm{H}), 4.08-3.99(\mathrm{~m}, 1 \mathrm{H})$, 
$3.77(\mathrm{td}, J=7.6,4.0 \mathrm{~Hz}, 1 \mathrm{H}), 2.40(\mathrm{~s}, 3 \mathrm{H}), 2.38-2.31(\mathrm{~m}, 4 \mathrm{H}), 2.19-2.10(\mathrm{~m}, 1 \mathrm{H}), 1.98-1.90$ (m, 1H), $1.89-1.78(\mathrm{~m}, 1 \mathrm{H}) .{ }^{13} \mathrm{C}$ NMR $\left(100 \mathrm{MHz}, \mathrm{CDCl}_{3}\right) \delta 159.8,152.9,151.2,140.9,134.7$, $128.7,128.7,128.5,128.3,125.3,124.6,124.2,124.1,118.3,76.5,69.5,30.2,27.5,20.3$, 11.5. HRMS (ESI): $m / z[M+H]^{+}$calculated for $\mathrm{C}_{21} \mathrm{H}_{21} \mathrm{O}_{3}: 321.1485$, found: 321.1484 .

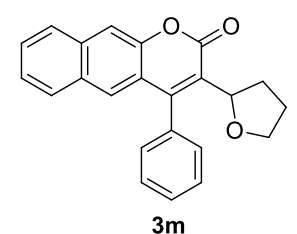

Compound $3 \mathrm{~m}$ was obtained as pale yellow oil in $70 \%$ yield according to the general procedure. ${ }^{1} \mathrm{H}$ NMR $\left(400 \mathrm{MHz}, \mathrm{CDCl}_{3}\right) \delta 8.65-8.58(\mathrm{~m}, 1 \mathrm{H}), 7.82(\mathrm{dd}, J=6.3,2.8 \mathrm{~Hz}, 1 \mathrm{H})$, $7.68-7.59(\mathrm{~m}, 2 \mathrm{H}), 7.58-7.48(\mathrm{~m}, 4 \mathrm{H}), 7.40-7.34(\mathrm{~m}, 1 \mathrm{H}), 7.26-7.21(\mathrm{~m}, 1 \mathrm{H}), 6.95(\mathrm{~d}, J=$ $8.8 \mathrm{~Hz}, 1 \mathrm{H}), 4.70(\mathrm{t}, J=8.1 \mathrm{~Hz}, 1 \mathrm{H}), 4.15-4.02(\mathrm{~m}, 1 \mathrm{H}), 3.81(\mathrm{td}, J=7.7,3.9 \mathrm{~Hz}, 1 \mathrm{H}), 2.43(\mathrm{dq}$, $J=11.6,8.7 \mathrm{~Hz}, 1 \mathrm{H}), 2.23-2.11(\mathrm{~m}, 1 \mathrm{H}), 2.05-1.94(\mathrm{~m}, 1 \mathrm{H}), 1.92-1.80(\mathrm{~m}, 1 \mathrm{H}) .{ }^{13} \mathrm{C}$ NMR $\left(100 \mathrm{MHz}, \mathrm{CDCl}_{3}\right) \delta 159.4,153.3,150.0,134.6,134.4,128.9,128.7,128.7,128.6,128.5,128.3$, 127.6, 127.0, 125.3, 123.6, 123.2, 122.9, 122.6, 115.5, 76.56, 69.6, 30.1, 27.5. HRMS (ESI): $m / z[\mathrm{M}+\mathrm{H}]^{+}$calculated for $\mathrm{C}_{23} \mathrm{H}_{19} \mathrm{O}_{3}: 343.1329$, found: 343.1328 .

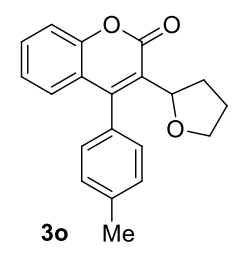

Compound 30 was obtained as a white solid in $63 \%$ yield according to the general procedure. Mp: $144-146^{\circ} \mathrm{C}^{1}{ }^{1} \mathrm{H}$ NMR $\left(400 \mathrm{MHz}, \mathrm{CDCl}_{3}\right) \delta 7.46$ (ddd, $J=8.6,7.3,1.6 \mathrm{~Hz}, 1 \mathrm{H}$ ), $7.36-7.29$ (m, 3H), $7.22(\mathrm{dd}, J=7.6,1.5 \mathrm{~Hz}, 1 \mathrm{H}), 7.14-7.06(\mathrm{~m}, 2 \mathrm{H}), 7.00(\mathrm{dd}, J=8.0,1.5 \mathrm{~Hz}, 1 \mathrm{H}), 4.68-$ $4.62(\mathrm{~m}, 1 \mathrm{H}), 4.07(\mathrm{td}, J=8.1,6.3 \mathrm{~Hz}, 1 \mathrm{H}), 3.79(\mathrm{td}, J=7.6,3.9 \mathrm{~Hz}, 1 \mathrm{H}), 2.45(\mathrm{~s}, 3 \mathrm{H}), 2.38$ (ddd, $J=17.3,11.6,8.7 \mathrm{~Hz}, 1 \mathrm{H}), 2.22-2.10(\mathrm{~m}, 1 \mathrm{H}), 1.99-1.90(\mathrm{~m}, 1 \mathrm{H}), 1.84(\mathrm{ddt}, J=16.9,11.8$, $8.2 \mathrm{~Hz}, 1 \mathrm{H}) .{ }^{13} \mathrm{C}$ NMR $\left(100 \mathrm{MHz}, \mathrm{CDCl}_{3}\right) \delta 159.4,153.0,152.7,138.6,131.2,129.4,129.1$, 128.6, 128.2, 127.8, 125.8, 123.8, 120.7, 116.4, 76.5, 69.5, 30.1, 27.5, 21.3. HRMS (ESI): $m / z$ $[\mathrm{M}+\mathrm{H}]^{+}$calculated for $\mathrm{C}_{20} \mathrm{H}_{19} \mathrm{O}_{3}: 307.1329$, found: 307.1327 . 


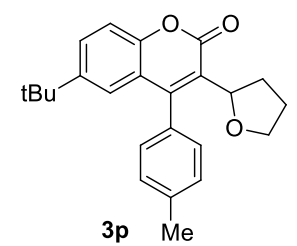

Compound $3 p$ was obtained as a white solid in $66 \%$ yield according to the general procedure. Mp: $155-157^{\circ} \mathrm{C} .{ }^{1} \mathrm{H}$ NMR $\left(400 \mathrm{MHz}, \mathrm{CDCl}_{3}\right) \delta 7.35(\mathrm{~d}, J=1.8 \mathrm{~Hz}, 1 \mathrm{H}), 7.30(\mathrm{t}, J=8.1 \mathrm{~Hz}, 2 \mathrm{H})$, $7.21(\mathrm{dd}, J=7.6,1.5 \mathrm{~Hz}, 1 \mathrm{H}), 7.14(\mathrm{dd}, J=8.4,1.9 \mathrm{~Hz}, 1 \mathrm{H}), 7.06$ (dd, $J=7.6,1.5 \mathrm{~Hz}, 1 \mathrm{H}$ ), 6.92 $(\mathrm{d}, J=8.4 \mathrm{~Hz}, 1 \mathrm{H}), 4.69-4.60(\mathrm{~m}, 1 \mathrm{H}), 4.07(\mathrm{td}, J=8.0,6.4 \mathrm{~Hz}, 1 \mathrm{H}), 3.78(\mathrm{td}, J=7.6,4.0 \mathrm{~Hz}$, 1H), $2.45(\mathrm{~s}, 3 \mathrm{H}), 2.36(\mathrm{ddd}, J=17.1,11.5,8.7 \mathrm{~Hz}, 1 \mathrm{H}), 2.20-2.10(\mathrm{~m}, 1 \mathrm{H}), 1.98-1.89(\mathrm{~m}$, 1H), $1.83(\mathrm{ddt}, J=16.8,11.7,8.2 \mathrm{~Hz}, 1 \mathrm{H}), 1.32(\mathrm{~s}, 9 \mathrm{H}) .{ }^{13} \mathrm{C}$ NMR $\left(100 \mathrm{MHz}, \mathrm{CDCl}_{3}\right) \delta 159.7$, $155.6,153.0,152.6,138.5,131.3,129.4,129.0,128.6,128.2,127.3,124.7,121.3,118.2$, 113.2, 76.5, 69.5, 35.1, 31.0, 30.1, 27.4, 21.3. HRMS (ESI): $\mathrm{m} / \mathrm{z}[\mathrm{M}+\mathrm{H}]^{+}$calculated for $\mathrm{C}_{24} \mathrm{H}_{27} \mathrm{O}_{3}$ : 363.1955, found: 363.1954 .<smiles>O=c1oc2ccc(Br)cc2c(C2CCCO2)c1-c1ccc(Cl)cc1</smiles>

Compound 3q was obtained as a white solid in $71 \%$ yield according to the general procedure. Mp: $192-193^{\circ} \mathrm{C} .{ }^{1} \mathrm{H}$ NMR $\left(400 \mathrm{MHz}, \mathrm{CDCl}_{3}\right) \delta 7.49$ (ddd, $\left.J=8.3,6.2,2.1 \mathrm{~Hz}, 2 \mathrm{H}\right), 7.37(\mathrm{~d}, J=$ $1.8 \mathrm{~Hz}, 1 \mathrm{H}), 7.29-7.24(\mathrm{~m}, 1 \mathrm{H}), 7.16(\mathrm{td}, J=8.6,2.2 \mathrm{~Hz}, 2 \mathrm{H}), 6.86(\mathrm{~d}, J=8.4 \mathrm{~Hz}, 1 \mathrm{H}), 4.66-$ $4.58(\mathrm{~m}, 1 \mathrm{H}), 3.99(\mathrm{td}, J=8.0,6.5 \mathrm{~Hz}, 1 \mathrm{H}), 3.77(\mathrm{td}, J=7.6,4.1 \mathrm{~Hz}, 1 \mathrm{H}), 2.32(\mathrm{dq}, J=11.5,8.6$ $\mathrm{Hz}, 1 \mathrm{H}), 2.19-2.07(\mathrm{~m}, 1 \mathrm{H}), 2.00-1.91(\mathrm{~m}, 1 \mathrm{H}), 1.91-1.78(\mathrm{~m}, 1 \mathrm{H}), 1.33(\mathrm{~s}, 9 \mathrm{H}) .{ }^{13} \mathrm{C} N M R$ $\left(100 \mathrm{MHz}, \mathrm{CDCl}_{3}\right) \delta 159.5,156.0,153.0,151.0,134.7,132.8,130.1,129.7,129.0,128.7,126.9$ $125.2,121.5,117.7,113.4,76.4,69.5,35.1,31.0,30.3,27.3$. HRMS (ESI): $m / z[M+H]^{+}$ calculated for $\mathrm{C}_{23} \mathrm{H}_{24} \mathrm{ClO}_{3}: 383.1408$, found: 383.1406 .<smiles>COc1ccc(-c2c(C3CCCO3)c(=O)oc3ccc(Br)cc23)cc1</smiles>

Compound $3 \mathrm{r}$ was obtained as a white solid in $62 \%$ yield according to the general procedure. Mp: $140-142^{\circ} \mathrm{C} .{ }^{1} \mathrm{H}$ NMR $\left(400 \mathrm{MHz}, \mathrm{CDCl}_{3}\right) \delta 7.35(\mathrm{~d}, J=1.8 \mathrm{~Hz}, 1 \mathrm{H}), 7.28-7.23(\mathrm{~m}, 1 \mathrm{H}), 7.16$ $(\mathrm{dd}, J=8.4,1.9 \mathrm{~Hz}, 1 \mathrm{H}), 7.13-7.08(\mathrm{~m}, 1 \mathrm{H}), 7.06-6.99(\mathrm{~m}, 2 \mathrm{H}), 6.96(\mathrm{~d}, J=8.4 \mathrm{~Hz}, 1 \mathrm{H}), 4.71$ 
$-4.64(\mathrm{~m}, 1 \mathrm{H}), 4.08(\mathrm{td}, J=8.0,6.4 \mathrm{~Hz}, 1 \mathrm{H}), 3.89(\mathrm{~s}, 3 \mathrm{H}), 3.79(\mathrm{td}, J=7.6,4.0 \mathrm{~Hz}, 1 \mathrm{H}), 2.37(\mathrm{dq}$, $J=11.1,8.6 \mathrm{~Hz}, 1 \mathrm{H}), 2.21-2.10(\mathrm{~m}, 1 \mathrm{H}), 1.98-1.89(\mathrm{~m}, 1 \mathrm{H}), 1.88-1.78(\mathrm{~m}, 1 \mathrm{H}), 1.33(\mathrm{~s}, 9 \mathrm{H})$. ${ }^{13} \mathrm{C}$ NMR $\left(100 \mathrm{MHz}, \mathrm{CDCl}_{3}\right) \delta 159.8,155.6,153.0,152.4,130.0,129.7,127.3,126.4,124.9$, 121.3, 118.3, 114.0, 113.3, 76.5, 69.5, 55.3, 35.1, 31.0, 30.1, 27.5. HRMS (ESI): $m / z[M+H]^{+}$ calculated for $\mathrm{C}_{24} \mathrm{H}_{27} \mathrm{O}_{4}: 379.1904$, found: 379.1906 .

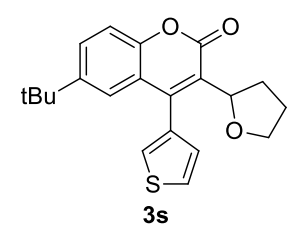

Compound 3s was obtained as pale yellow oil in $75 \%$ yield according to the general procedure. ${ }^{1} \mathrm{H}$ NMR $\left(400 \mathrm{MHz}, \mathrm{CDCl}_{3}\right) \delta 7.50(\mathrm{dd}, \mathrm{J}=4.9,3.0 \mathrm{~Hz}, 1 \mathrm{H}), 7.35(\mathrm{~d}, \mathrm{~J}=1.8 \mathrm{~Hz}, 1 \mathrm{H})$, $7.30(\mathrm{~s}, 1 \mathrm{H}), 7.19(\mathrm{dd}, \mathrm{J}=8.4,1.9 \mathrm{~Hz}, 1 \mathrm{H}), 7.08(\mathrm{~s}, 1 \mathrm{H}), 7.03(\mathrm{~d}, \mathrm{~J}=8.4 \mathrm{~Hz}, 1 \mathrm{H}), 4.78-4.70(\mathrm{~m}$, $1 \mathrm{H}), 4.16-4.00(\mathrm{~m}, 1 \mathrm{H}), 3.81(\mathrm{td}, \mathrm{J}=7.6,4.1 \mathrm{~Hz}, 1 \mathrm{H}), 2.35(\mathrm{dq}, \mathrm{J}=11.2,8.6 \mathrm{~Hz}, 1 \mathrm{H}), 2.22-$ $2.11(\mathrm{~m}, 1 \mathrm{H}), 2.02-1.93(\mathrm{~m}, 1 \mathrm{H}), 1.93-1.81(\mathrm{~m}, 1 \mathrm{H}), 1.33(\mathrm{~s}, 9 \mathrm{H}) .{ }^{13} \mathrm{C} \mathrm{NMR}\left(100 \mathrm{MHz}, \mathrm{CDCl}_{3}\right)$ $\delta 159.6,155.8,152.8,148.0,134.0,128.5,126.9,126.4,125.7,121.5,118.0,113.3,76.5$, 69.5, 35.1, 31.0, 30.2, 27.4. HRMS (ESI): $\mathrm{m} / \mathrm{z}[\mathrm{M}+\mathrm{H}]^{+}$calculated for $\mathrm{C}_{21} \mathrm{H}_{23} \mathrm{O}_{3} \mathrm{~S}: 355.1362$, found: 355.1361 .<smiles>CCOC(C)c1c(-c2ccccc2)c2cc(Br)ccc2oc1=O</smiles>

Compound 3t was obtained as ropy oil in $54 \%$ yield according to the general procedure. ${ }^{1} \mathbf{H}$ NMR $\left(400 \mathrm{MHz}, \mathrm{CDCl}_{3}\right) \delta 7.55-7.47(\mathrm{~m}, 3 \mathrm{H}), 7.36(\mathrm{~d}, J=1.8 \mathrm{~Hz}, 1 \mathrm{H}), 7.27(\mathrm{~s}, 1 \mathrm{H}), 7.20-7.14$ $(\mathrm{m}, 2 \mathrm{H}), 6.85(\mathrm{~d}, J=8.4 \mathrm{~Hz}, 1 \mathrm{H}), 4.36(\mathrm{q}, J=6.6 \mathrm{~Hz}, 1 \mathrm{H}), 3.36(\mathrm{dq}, J=9.0,7.0 \mathrm{~Hz}, 1 \mathrm{H}), 3.20(\mathrm{dq}$, $J=9.1,7.0 \mathrm{~Hz}, 1 \mathrm{H}), 1.52(\mathrm{~d}, J=6.7 \mathrm{~Hz}, 3 \mathrm{H}), 1.33(\mathrm{~s}, 9 \mathrm{H}), 1.04(\mathrm{t}, J=7.0 \mathrm{~Hz}, 3 \mathrm{H}) .{ }^{13} \mathrm{C}$ NMR $(100$ $\left.\mathrm{MHz}, \mathrm{CDCl}_{3}\right) \delta 159.6,155.8,153.0,152.0,134.5,128.6,128.5,128.3,128.0,127.2,125.2$, 121.4, 118.1, 113.2, 72.9, 64.1, 35.1, 31.0, 20.0, 15.2. HRMS (ESI): $m / z[M+H]^{+}$calculated for $\mathrm{C}_{23} \mathrm{H}_{27} \mathrm{O}_{3}: 351.1955$, found: 351.1954 .

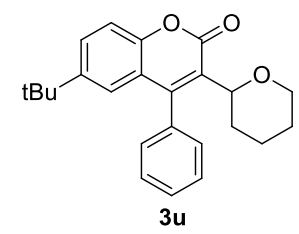


Compound $3 \mathbf{u}$ was obtained as colorless oil in $51 \%$ yield according to the general procedure. ${ }^{1} \mathrm{H}$ NMR $\left(400 \mathrm{MHz}, \mathrm{CDCl}_{3}\right) \delta 7.59-7.46(\mathrm{~m}, 3 \mathrm{H}), 7.36(\mathrm{~d}, J=1.8 \mathrm{~Hz}, 1 \mathrm{H}), 7.34-7.29(\mathrm{~m}, 1 \mathrm{H})$, $7.23-7.19(\mathrm{~m}, 1 \mathrm{H}), 7.14(\mathrm{dd}, J=8.4,1.9 \mathrm{~Hz}, 1 \mathrm{H}), 6.87(\mathrm{~d}, J=8.4 \mathrm{~Hz}, 1 \mathrm{H}), 4.14(\mathrm{dd}, J=11.5$, $2.2 \mathrm{~Hz}, 1 \mathrm{H}$ ), $3.97(\mathrm{dd}, J=11.3,4.4 \mathrm{~Hz}, 1 \mathrm{H}$ ), $3.29-3.17(\mathrm{~m}, 1 \mathrm{H}), 2.35$ (ddd, $J=24.6,12.9,4.0$ $\mathrm{Hz}, 1 \mathrm{H}), 1.81(\mathrm{~d}, J=12.8 \mathrm{~Hz}, 1 \mathrm{H}), 1.66-1.52(\mathrm{~m}, 3 \mathrm{H}), 1.45(\mathrm{~d}, J=15.7 \mathrm{~Hz}, 1 \mathrm{H}), 1.32(\mathrm{~s}, 9 \mathrm{H}) .{ }^{13} \mathrm{C}$ NMR $\left(100 \mathrm{MHz}, \mathrm{CDCl}_{3}\right) \delta 160.0,155.9,153.0,152.5,134.6,128.6,128.5,128.2,128.1,127.4$, 124.7, 121.4, 118.1, 113.2, 76.6, 68.9, 35.1, 31.0, 28.6, 25.4, 23.7. HRMS (ESI): $m / z[M+H]^{+}$ calculated for $\mathrm{C}_{24} \mathrm{H}_{27} \mathrm{O}_{3}: 363.1955$, found: 363.1956 .

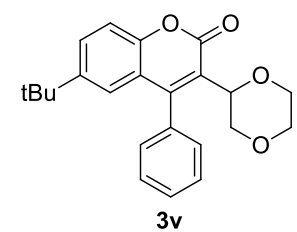

Compound $3 \mathbf{v}$ was obtained as ropy oil in $65 \%$ yield according to the general procedure. ${ }^{1} \mathbf{H}$ NMR $\left(400 \mathrm{MHz}, \mathrm{CDCl}_{3}\right) \delta 7.56-7.49(\mathrm{~m}, 3 \mathrm{H}), 7.37(\mathrm{~d}, J=1.8 \mathrm{~Hz}, 1 \mathrm{H}), 7.34-7.30(\mathrm{~m}, 1 \mathrm{H})$, $7.23-7.19(\mathrm{~m}, 1 \mathrm{H}), 7.17(\mathrm{dd}, J=8.5,1.9 \mathrm{~Hz}, 1 \mathrm{H}), 6.90(\mathrm{~d}, J=8.4 \mathrm{~Hz}, 1 \mathrm{H}), 4.43(\mathrm{dd}, J=10.3$, $2.5 \mathrm{~Hz}, 1 \mathrm{H}), 4.38-4.31(\mathrm{~m}, 1 \mathrm{H}), 3.78(\mathrm{dd}, J=11.4,2.1 \mathrm{~Hz}, 1 \mathrm{H}), 3.68(\mathrm{dd}, J=11.8,2.6 \mathrm{~Hz}, 1 \mathrm{H})$, $3.63-3.52(\mathrm{~m}, 3 \mathrm{H}), 1.32(\mathrm{~s}, 9 \mathrm{H}) .{ }^{13} \mathrm{C}$ NMR $\left(100 \mathrm{MHz}, \mathrm{CDCl}_{3}\right) \delta 159.9,156.6,155.1,153.2$, 134.1, 128.9, 128.7, 128.5, 128.4, 127.9, 127.6, 121.7, 120.8, 117.7, 113.3, 74.66, 68.1, 67.1, 66.0, 35.2, 31.0. HRMS (ESI): $m / z[M+H]^{+}$calculated for $\mathrm{C}_{23} \mathrm{H}_{25} \mathrm{O}_{3}: 365.1747$, found: 365.1749 .

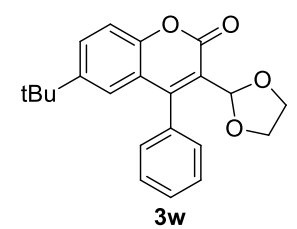

Compound $3 w$ was obtained as a white solid in $42 \%$ yield according to the general procedure. Mp: 65-67 ${ }^{\circ} \mathrm{C} .{ }^{1} \mathrm{H}$ NMR (400 MHz, $\left.\mathrm{CDCl}_{3}\right) \delta 7.55-7.44(\mathrm{~m}, 3 \mathrm{H}), 7.37$ (d, J=1.8 Hz, 1H), $7.34-$ $7.28(\mathrm{~m}, 2 \mathrm{H}), 7.18(\mathrm{dd}, J=8.5,1.8 \mathrm{~Hz}, 1 \mathrm{H}), 6.94(\mathrm{~d}, J=8.5 \mathrm{~Hz}, 1 \mathrm{H}), 5.69(\mathrm{~s}, 1 \mathrm{H}), 4.11(\mathrm{td}, J=$ 6.4, 4.0 Hz, 2H), $3.84(\mathrm{td}, J=6.3,4.0 \mathrm{~Hz}, 2 \mathrm{H}), 1.33(\mathrm{~s}, 9 \mathrm{H}) .{ }^{13} \mathrm{C}$ NMR $\left(100 \mathrm{MHz}, \mathrm{CDCl}_{3}\right) \delta$ 159.0, $156.8,154.9,153.5,133.3,128.8,128.7,128.4,127.5,121.6,120.5,117.7,113.5,100.8,66.3$, 35.2, 31.0. HRMS (ESI): $m / z[M+H]^{+}$calculated for $\mathrm{C}_{22} \mathrm{H}_{23} \mathrm{O}_{4}: 351.1591$, found: 351.1590 . 
<smiles>COCC(OC)c1c(-c2ccccc2)c2cc(CC(C)C)ccc2oc1=O</smiles><smiles>COCCOCc1c(-c2ccccc2)c2cc(C(C)(C)C)ccc2oc1=O</smiles>

Compound $\mathbf{3 x}$ and $3 \mathbf{x}^{\prime}$ was obtained in $60 \%$ total yield according to the general procedure. $3 x: 3 x^{\prime}=1: 0.65 .{ }^{1} \mathbf{H}$ NMR $\left(400 \mathrm{MHz}, \mathrm{CDCl}_{3}\right) \delta 7.55-7.47(\mathrm{~m}, 5 \mathrm{H}), 7.40-7.35(\mathrm{~m}, 3 \mathrm{H}), 7.27(\mathrm{~s}$, 1H), $7.26-7.24(m, 1 H), 7.18(d d d, J=8.4,4.5,1.9 \mathrm{~Hz}, 2 \mathrm{H}), 7.02(\mathrm{~d}, J=8.4 \mathrm{~Hz}, 0.65 \mathrm{H}), 6.88(\mathrm{~d}$, $J=8.4 \mathrm{~Hz}, 1 \mathrm{H}), 4.32(\mathrm{dd}, J=6.9,5.5 \mathrm{~Hz}, 1 \mathrm{H}), 4.24(\mathrm{~s}, 1 \mathrm{H}), 3.90(\mathrm{dd}, J=10.0,7.0 \mathrm{~Hz}, 1 \mathrm{H}), 3.78$ (dd, $J=10.0,5.4 \mathrm{~Hz}, 1 \mathrm{H}), 3.58(\mathrm{dd}, J=5.8,3.6 \mathrm{~Hz}, 1 \mathrm{H}), 3.49(\mathrm{dd}, J=5.9,3.6 \mathrm{~Hz}, 1 \mathrm{H}), 3.33(\mathrm{~s}$, $2 \mathrm{H}), 3.31(\mathrm{~s}, 3 \mathrm{H}), 3.20(\mathrm{~s}, 3 \mathrm{H}), 1.33(\mathrm{~d}, J=1.2 \mathrm{~Hz}, 15 \mathrm{H}) .{ }^{13} \mathrm{C}$ NMR $\left(100 \mathrm{MHz}, \mathrm{CDCl}_{3}\right) \delta 161.9$, $159.9,156.3,156.2,154.7,154.5,153.4,153.1,134.0,133.7,129.0,128.9,128.8,128.8$, $128.7,128.4,128.4,128.0,127.5,127.5,121.6,121.6,121.4,121.4,118.0,117.8,113.5$, 113.3, 78.1, 73.7, 71.8, 70.1, 65.7, 59.1, 58.9, 57.2, 35.1, 31.0. HRMS (ESI): $m / z[M+H]^{+}$ calculated for $\mathrm{C}_{23} \mathrm{H}_{27} \mathrm{O}_{3}: 367.1904$, found: 367.1908 .

Reference:

[1] Hua, H.-L.; He, Y.-T.; Qiu, Y.-F.; Li, Y.-X.; Song, B.; Gao, P.; Song, X.-R.; Guo, D.-H.; Liu, X.-Y.; Liang, Y.-M.Chem. Eur. J.2015, 21, 1468.

\section{Kinetic isotope effect experiment.}
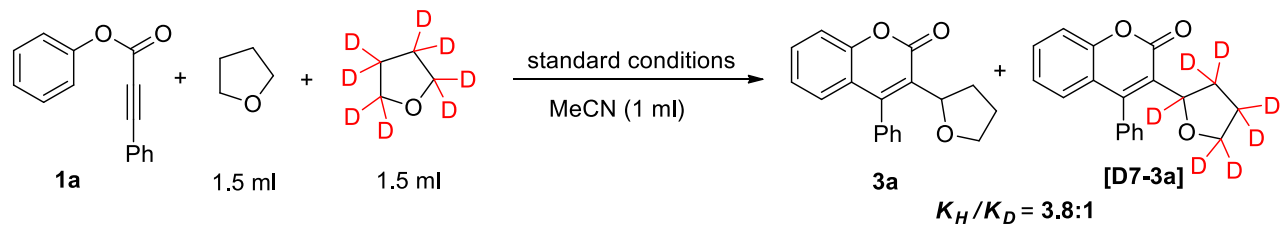

To a reaction tube equipped with a magnetic stir bar was added 1 a $(0.15 \mathrm{mmol}, 1.0$ equiv), $\mathrm{Ru}(\mathrm{bpy})_{3} \mathrm{Cl}_{2} \cdot 6 \mathrm{H}_{2} \mathrm{O}(5 \mathrm{~mol} \%, 6 \mathrm{mg})$, in mixed solvent of $\mathrm{MeCN}(1.0 \mathrm{~mL})$, THF $(1.5 \mathrm{~mL})$ and D8-THF (1.5 mL) was added $t$-BuOOH (5.5 M in decane) (0.6 mmol, 4.0 equiv). Then the reaction was irradiated with a $34 \mathrm{~W}$ blue LED strip and stirred at ambient temperature for 40 hours. The reaction was monitored by TLC to establish the consumption of starting material. After it was complete, the reaction mixture was concentrated under reduced pressure to give a residue which was purified by silica gel column chromatography to afford desired pure product. 


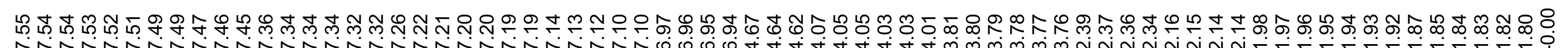

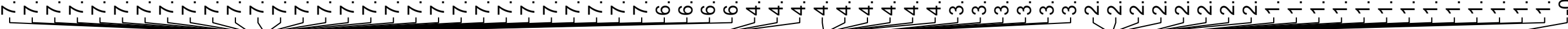
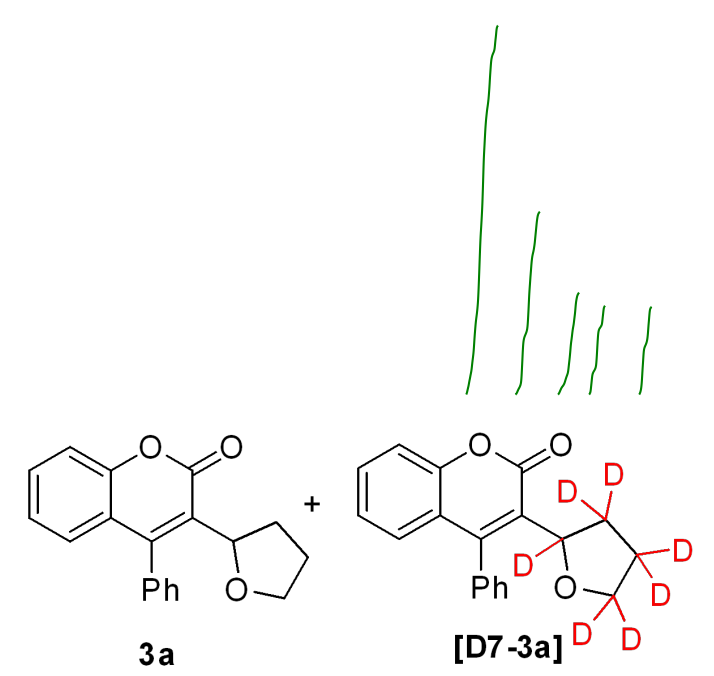

$3 a$
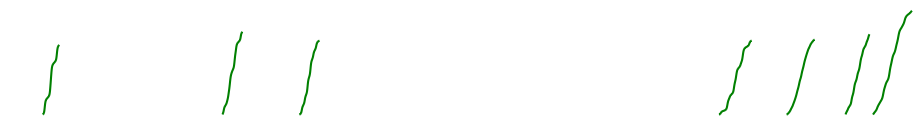

${ }^{1} \mathrm{H} \mathrm{NMR}\left(400 \mathrm{MHz}, \mathrm{CDCl}_{3}\right)$

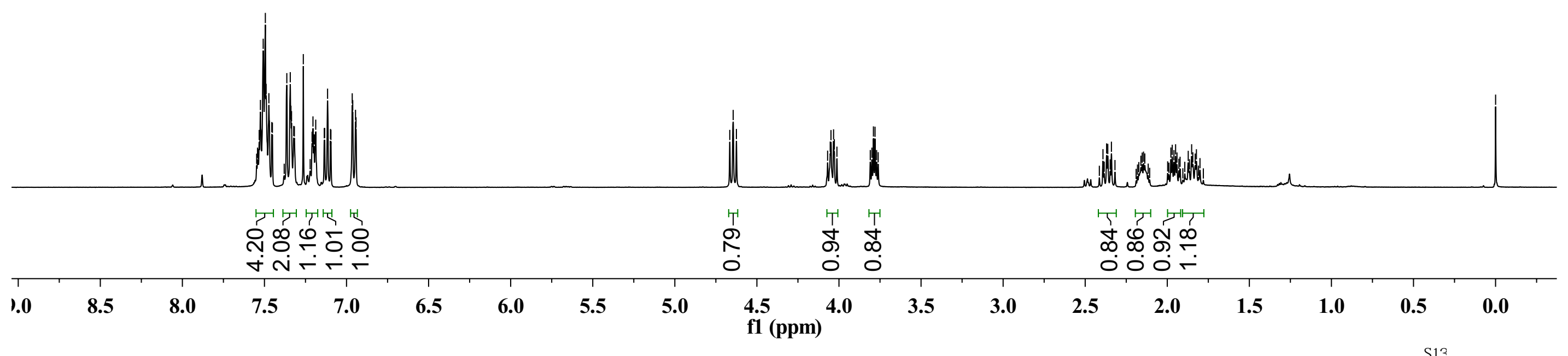



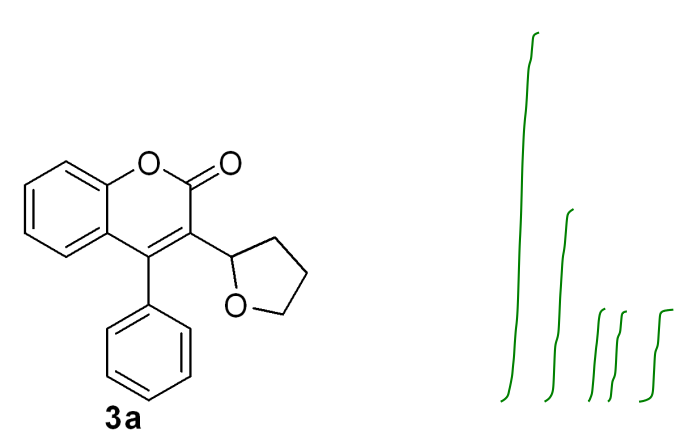

${ }^{1} \mathrm{H}$ NMR $\left(400 \mathrm{MHz}, \mathrm{CDCl}_{3}\right)$
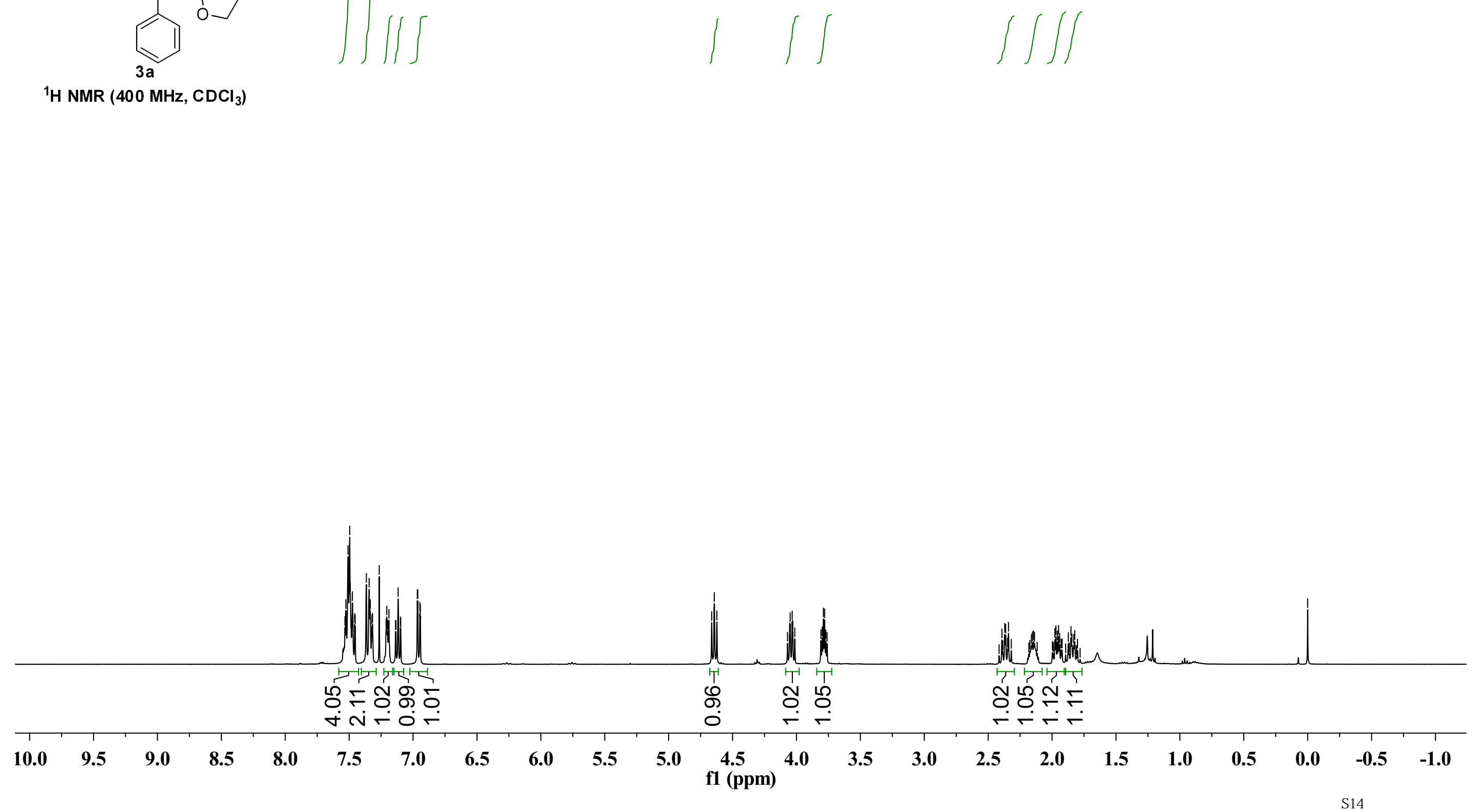


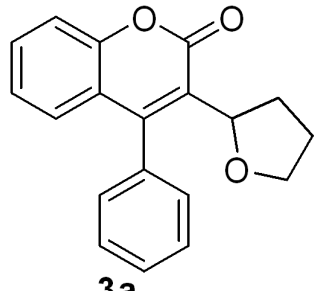

${ }^{13} \mathrm{C} \mathrm{NMR}\left(100 \mathrm{MHz}, \mathrm{CDCl}_{3}\right)$

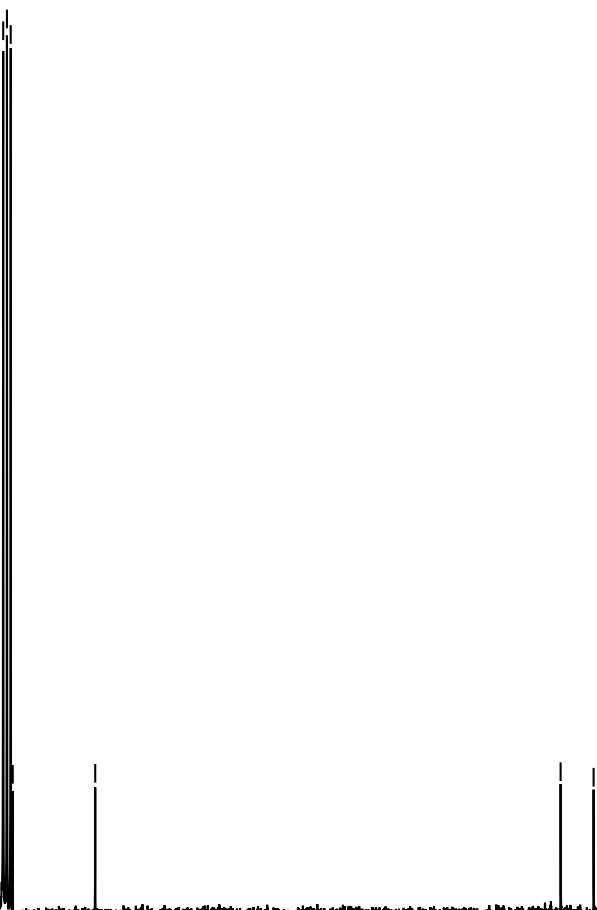

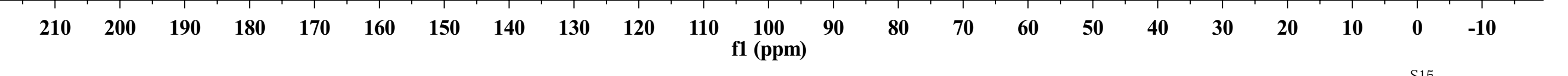




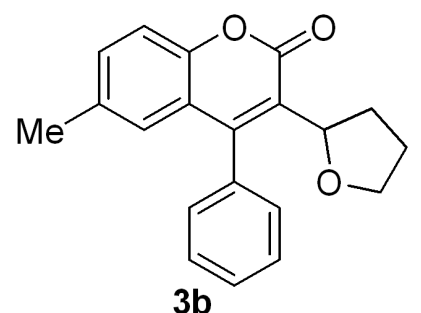

$3 b$
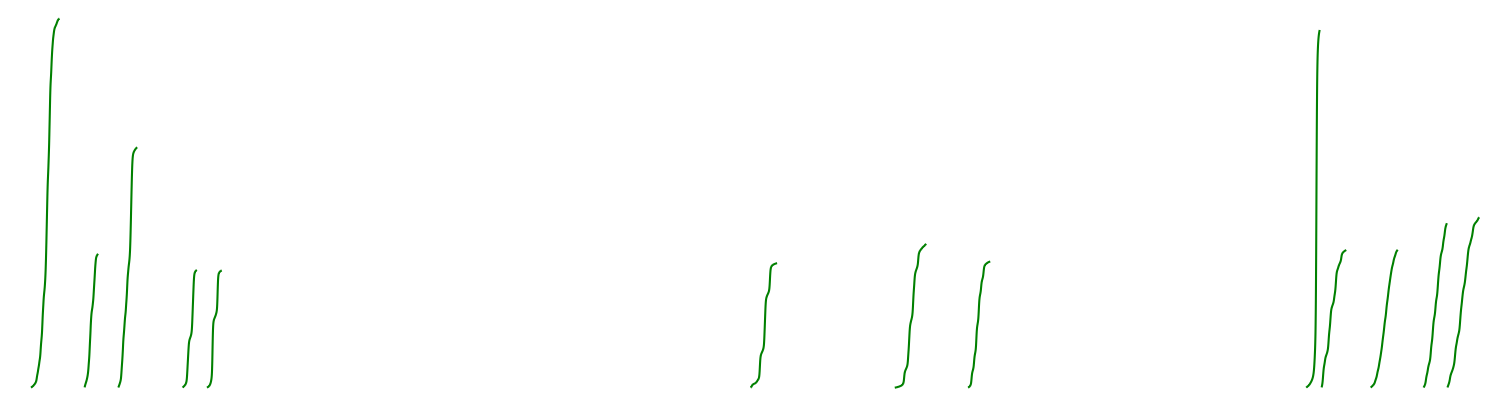

${ }^{1} \mathrm{H} \mathrm{NMR}\left(400 \mathrm{MHz}, \mathrm{CDCl}_{3}\right)$

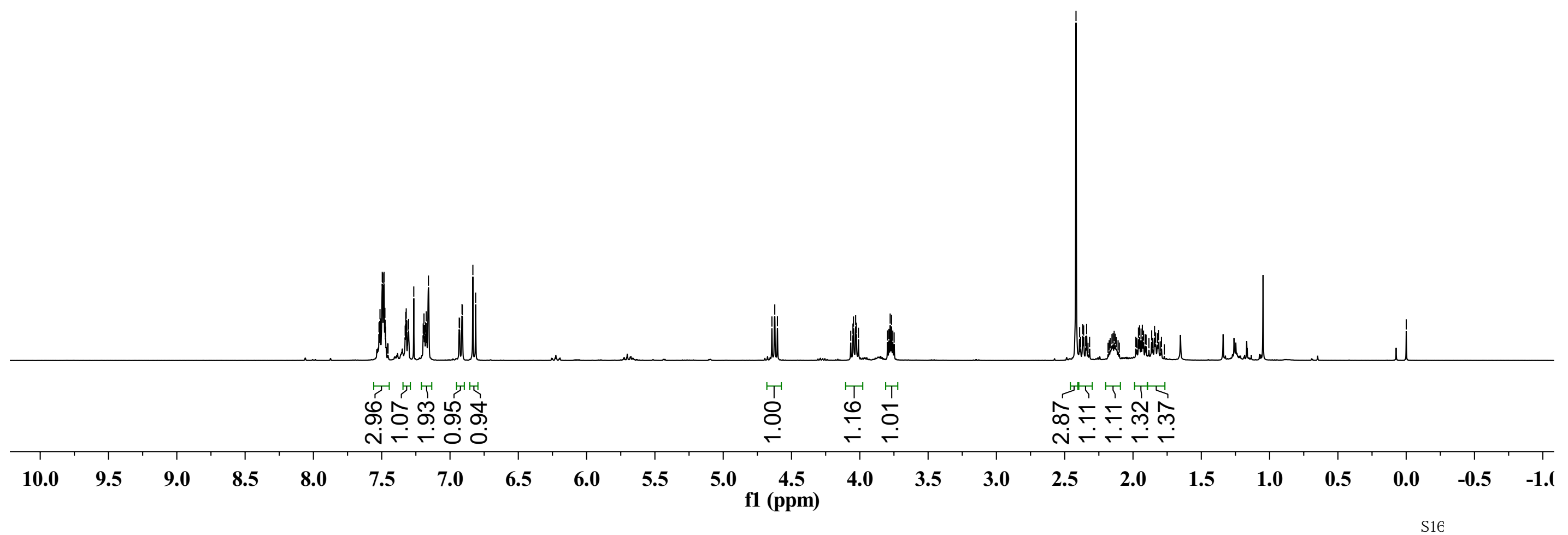




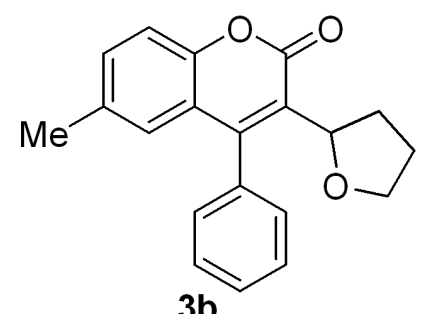

${ }^{13} \mathrm{C}$ NMR (100 MHz, CDCl 3 )

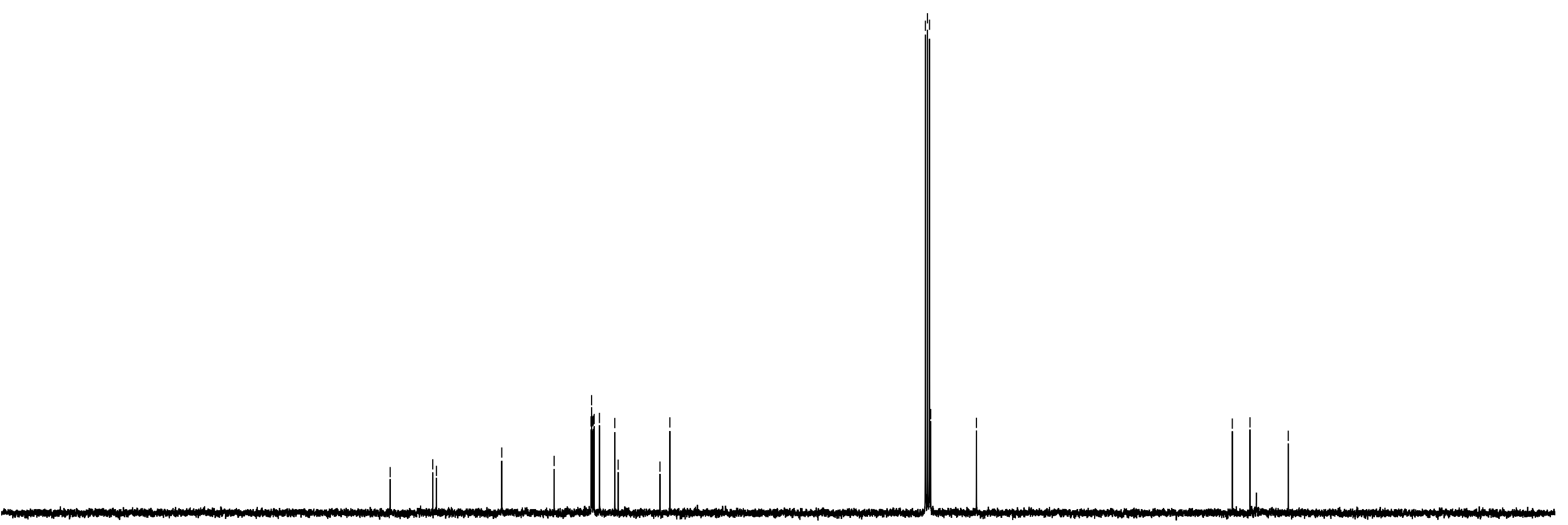

\begin{tabular}{|c|c|c|c|c|c|c|c|c|c|c|c|c|c|c|c|c|c|c|c|c|c|c|}
\hline 210 & 200 & 190 & 180 & 170 & 160 & 150 & 140 & 130 & 120 & 110 & $\begin{array}{c}100 \\
\text { f1 (ppm) }\end{array}$ & 90 & 80 & 70 & 60 & 50 & 40 & 30 & 20 & 10 & 0 & -10 \\
\hline
\end{tabular}



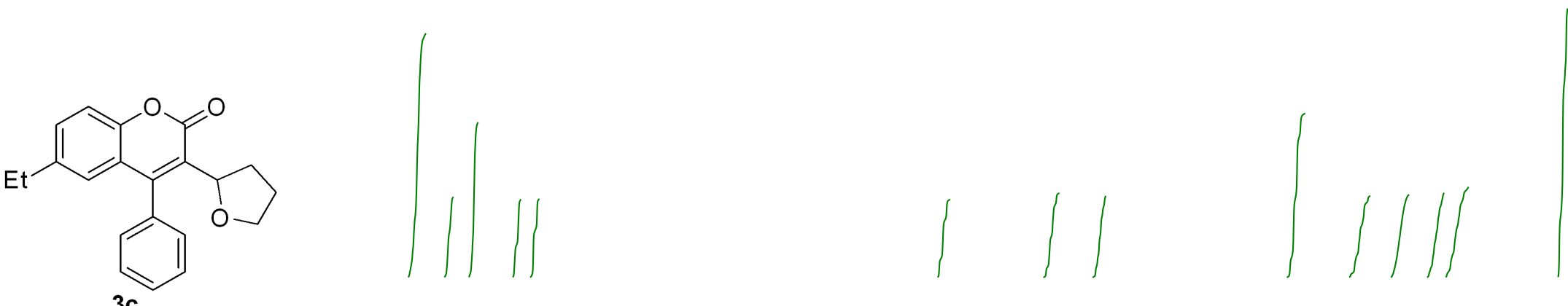

${ }^{1} \mathrm{H}$ NMR (400 MHz, $\mathrm{CDCl}_{3}$ )

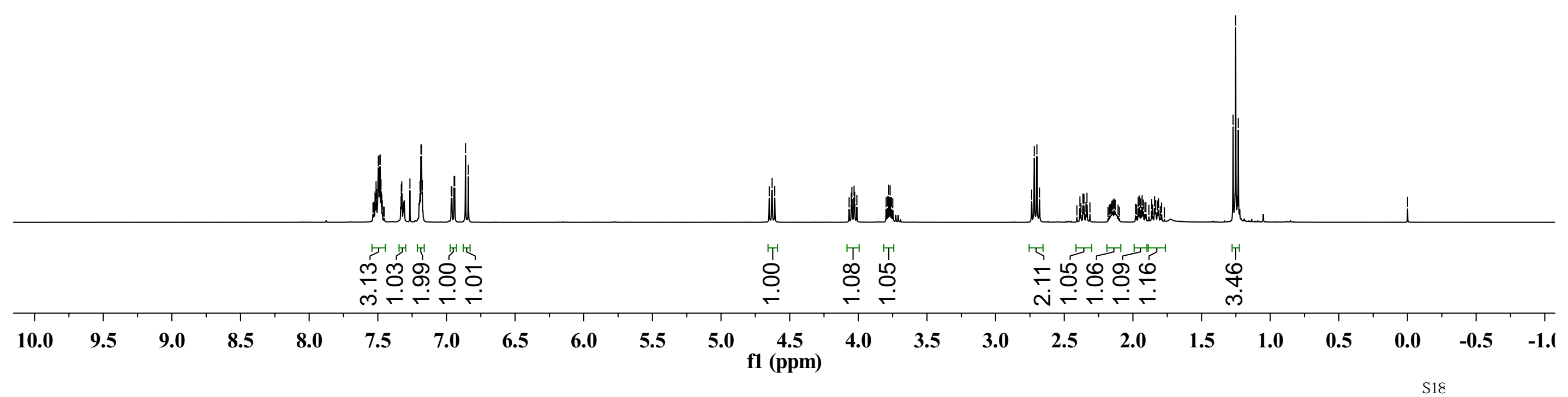




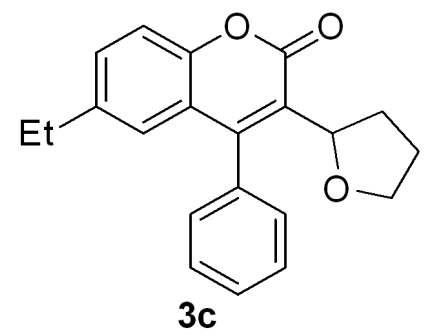

${ }^{13} \mathrm{C}$ NMR $\left(100 \mathrm{MHz}^{\mathrm{CDCl}}\right)_{3}$

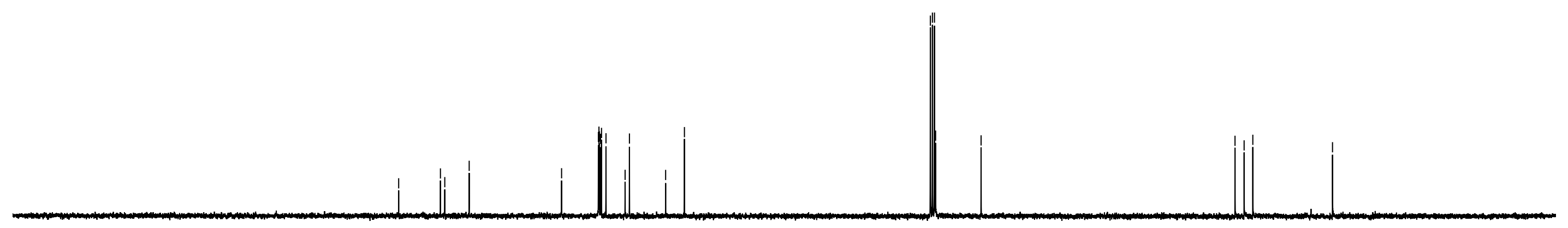



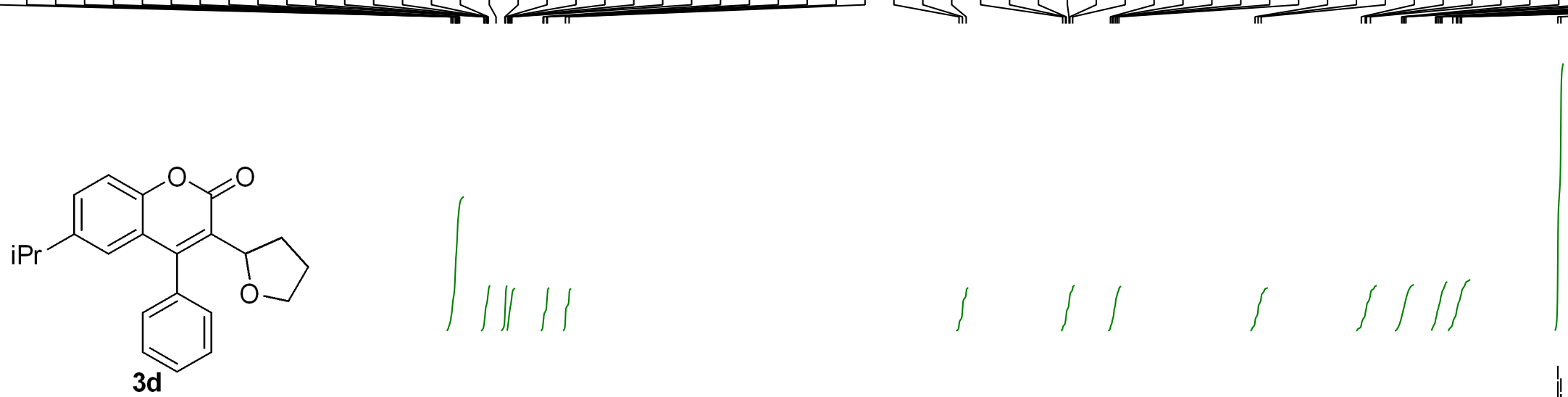

${ }^{1} \mathrm{H}$ NMR $\left(400 \mathrm{MHz}, \mathrm{CDCl}_{3}\right)$

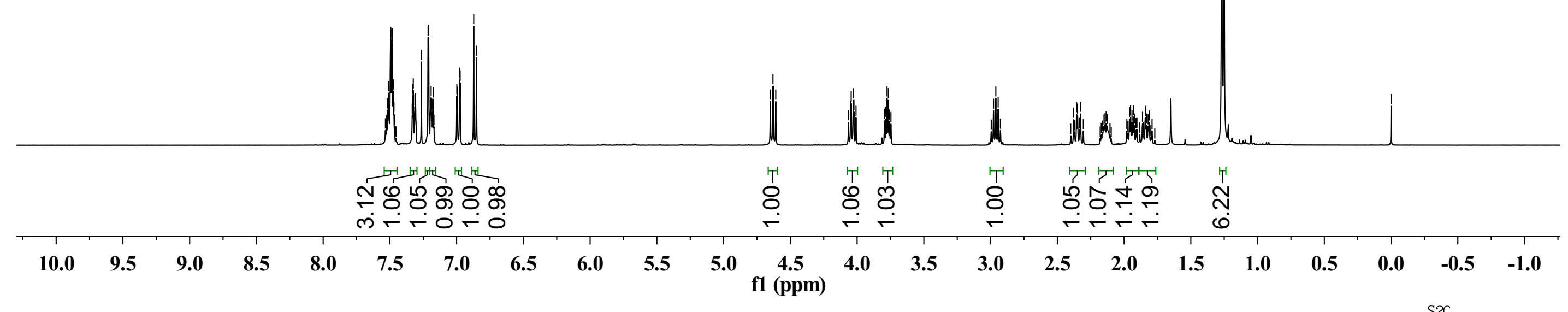




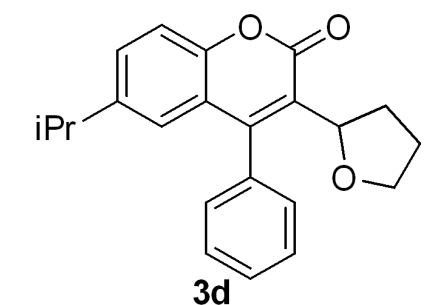

${ }^{13} \mathrm{C}$ NMR (100 MHz, $\left.\mathrm{CDCl}_{3}\right)$

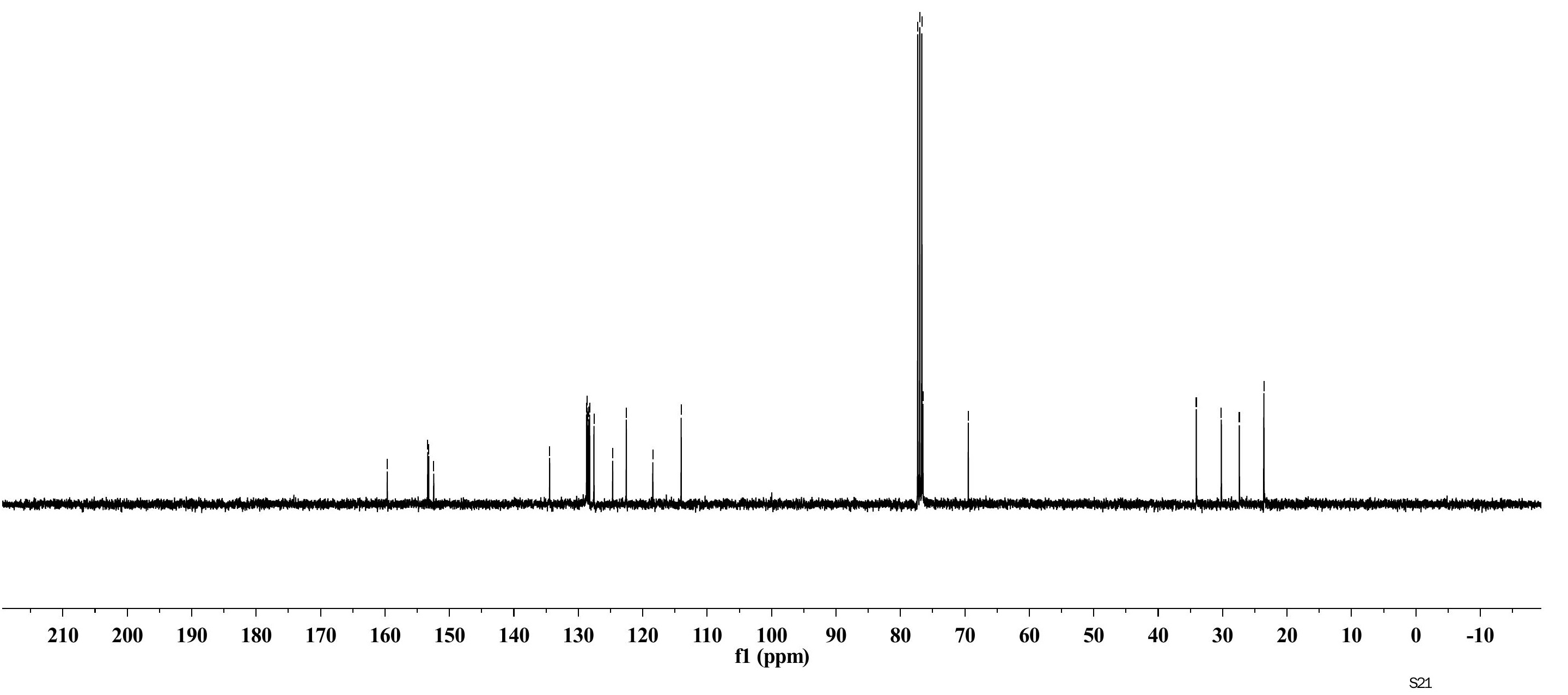



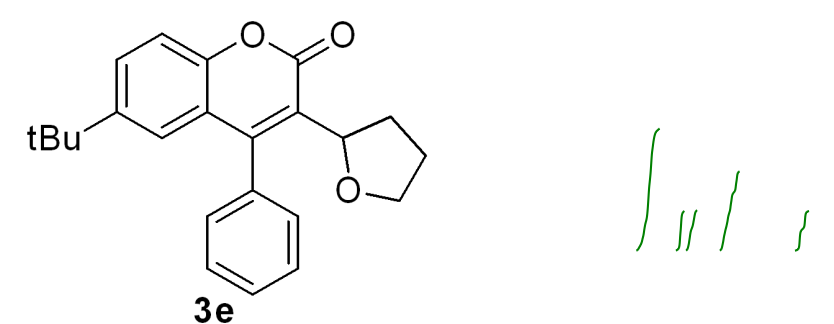

${ }^{1} \mathrm{H}$ NMR (400 MHz, $\left.\mathrm{CDCl}_{3}\right)$

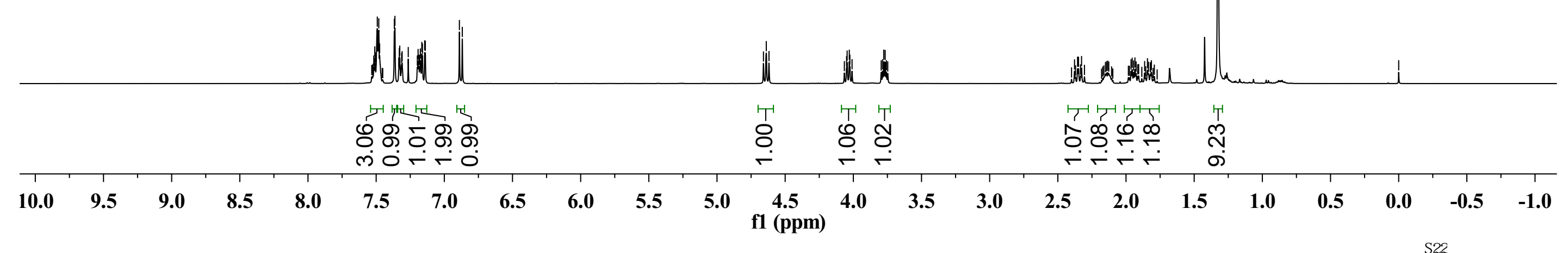




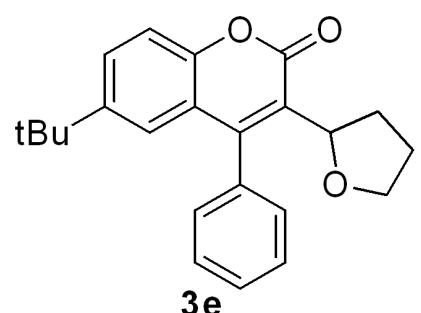

${ }^{13} \mathrm{C}$ NMR $\left(100 \mathrm{MHz}, \mathrm{CDCl}_{3}\right)$

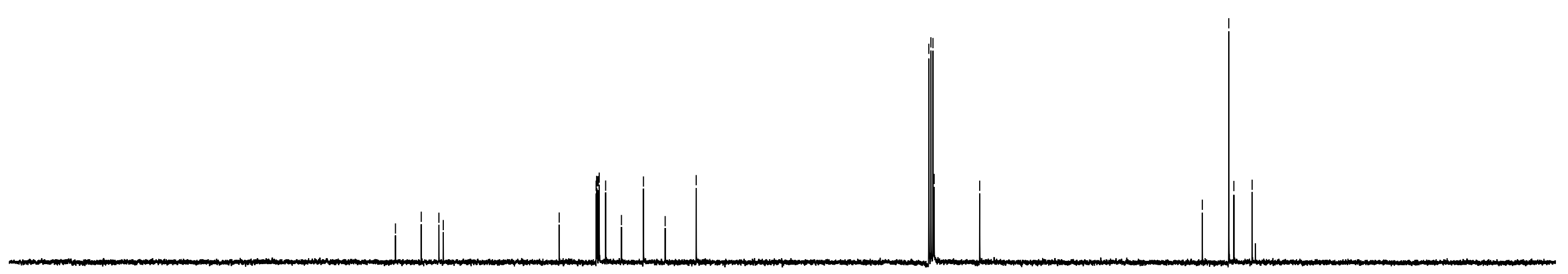

70



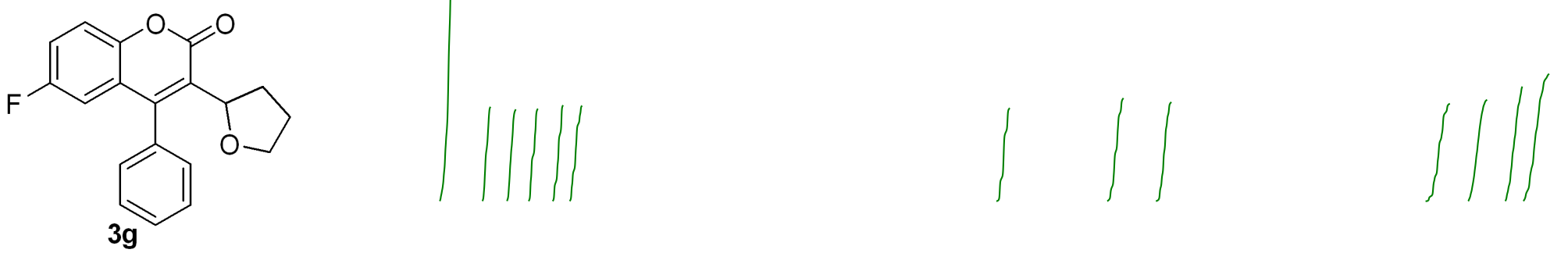

${ }^{1} \mathrm{H} \mathrm{NMR}\left(400 \mathrm{MHz}, \mathrm{CDCl}_{3}\right)$

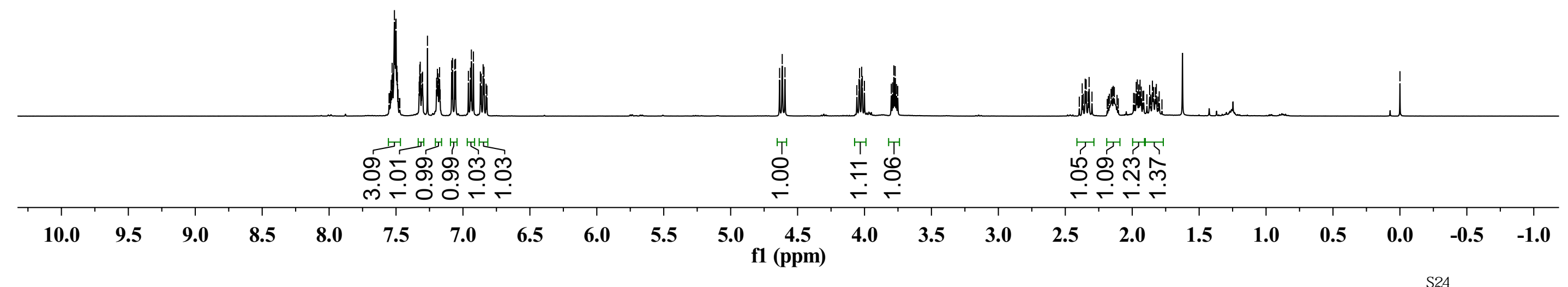




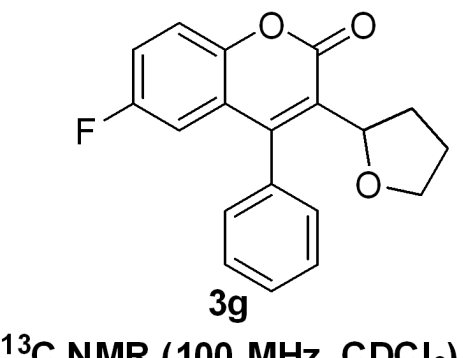

${ }^{13} \mathrm{C}$ NMR (100 MHz, CDCl 3 )

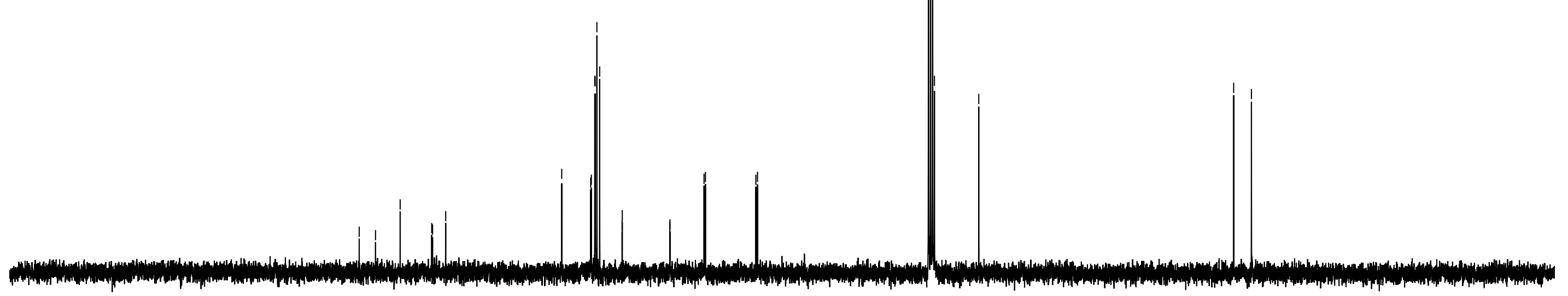




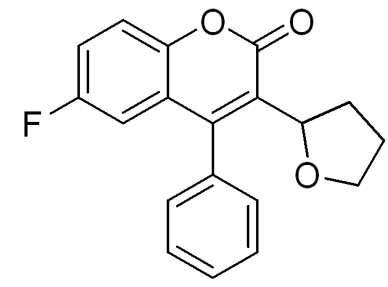

$3 \mathrm{~g}$

${ }^{19} \mathrm{~F}$ NMR $\left(376 \mathrm{MHz}, \mathrm{CDCl}_{3}\right)$

\begin{tabular}{rlllllllllllllllllllllllll}
\hline 10 & 0 & -10 & -20 & -30 & -40 & -50 & -60 & -70 & -80 & -90 & -100 & -110 & -120 & -130 & -140 & -150 & -160 & -170 & -180 & -190 & -200 & -210 & & &
\end{tabular}




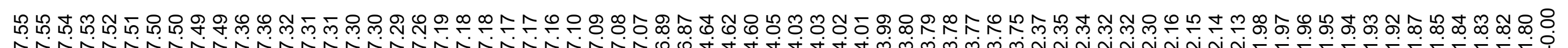

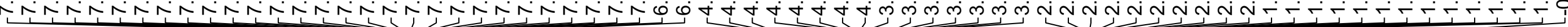
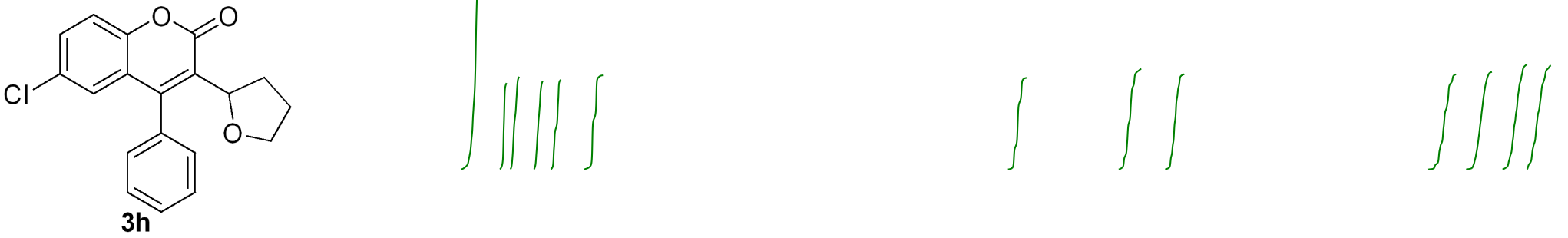

${ }^{1} \mathrm{H}$ NMR $\left(400 \mathrm{MHz}, \mathrm{CDCl}_{3}\right)$

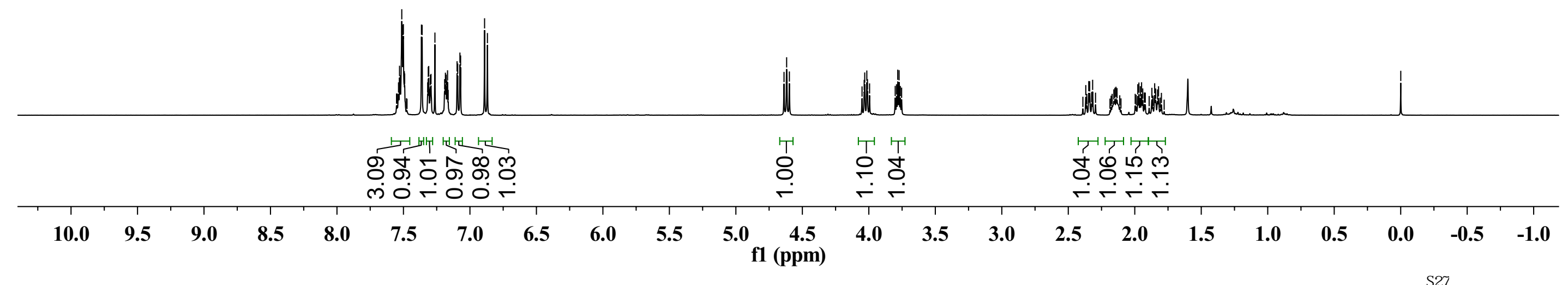




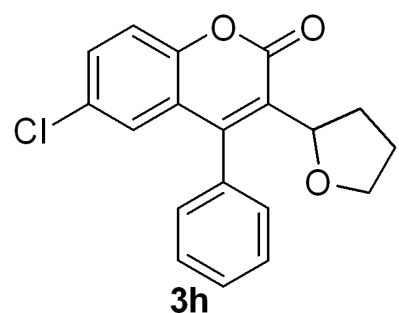

${ }^{13} \mathrm{C}$ NMR $\left(100 \mathrm{MHz}, \mathrm{CDCl}_{3}\right)$

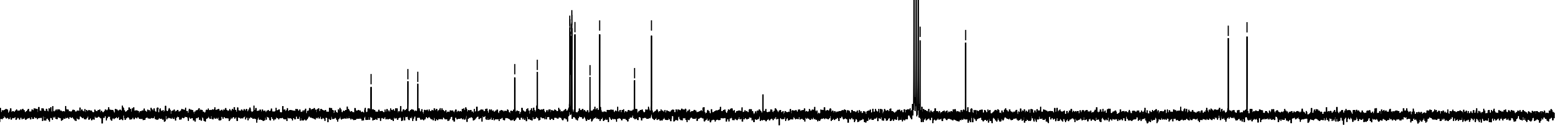

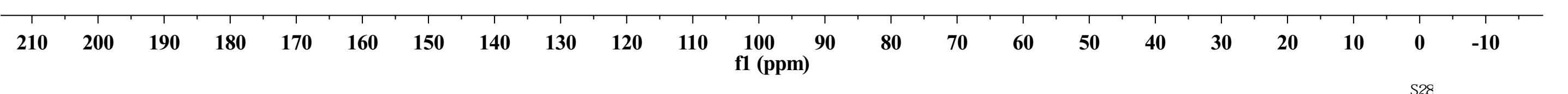



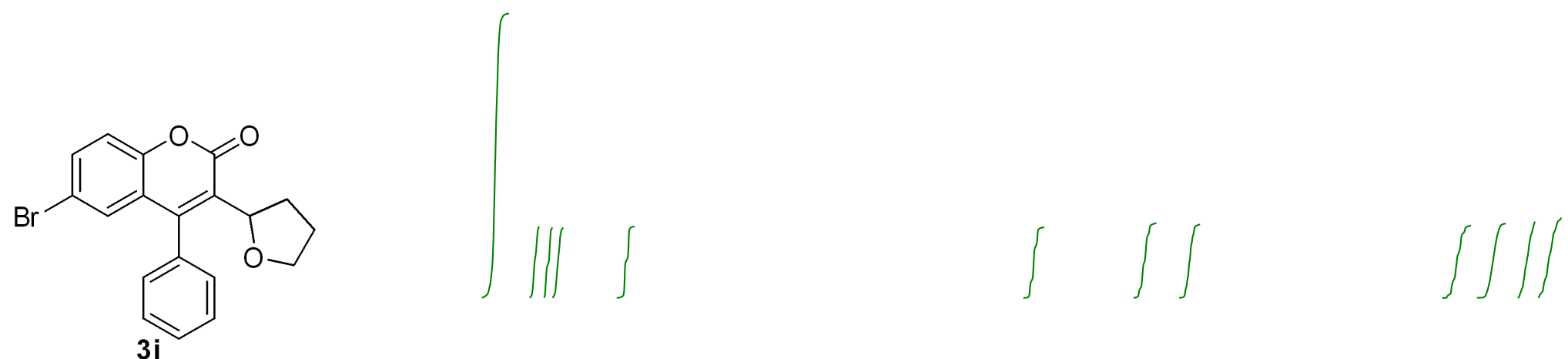

${ }^{1} \mathrm{H}$ NMR $\left(400 \mathrm{MHz}, \mathrm{CDCl}_{3}\right)$

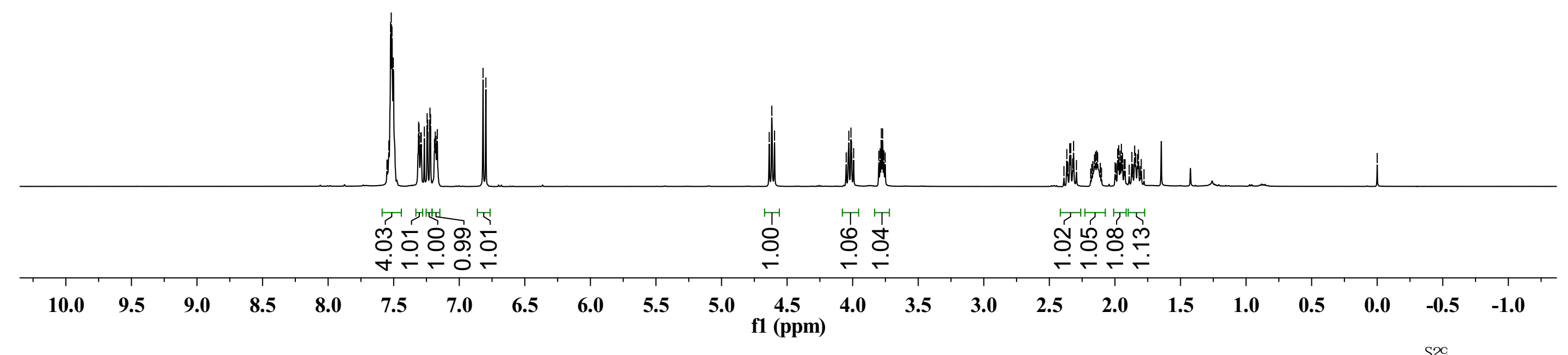




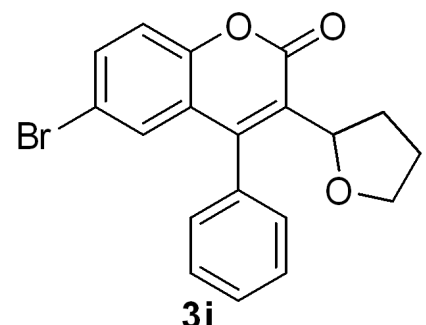

${ }^{13} \mathrm{C}$ NMR (100 MHz, $\mathrm{CDCl}_{3}$ )

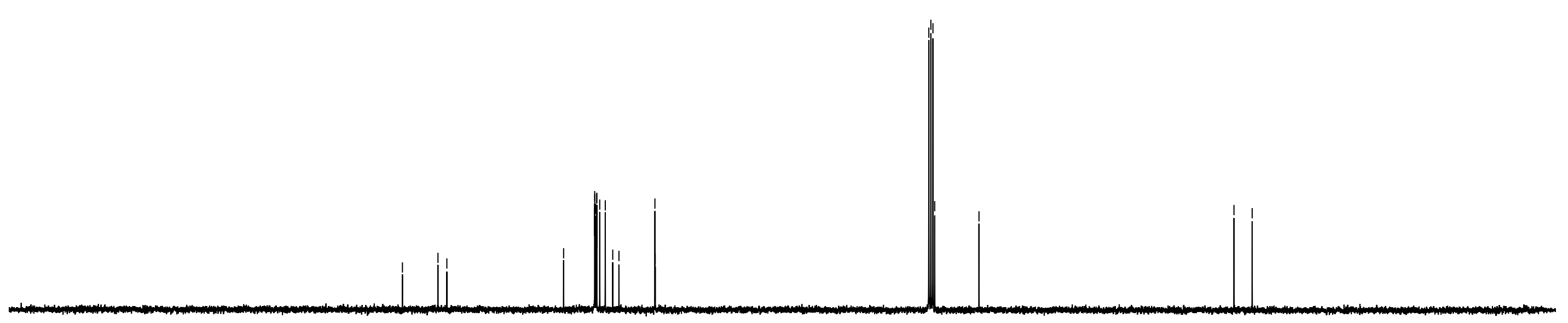




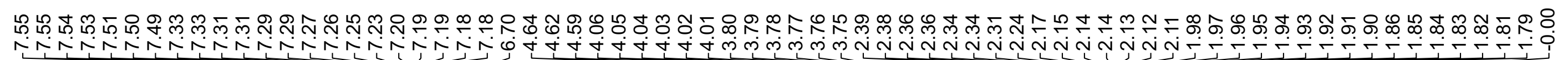
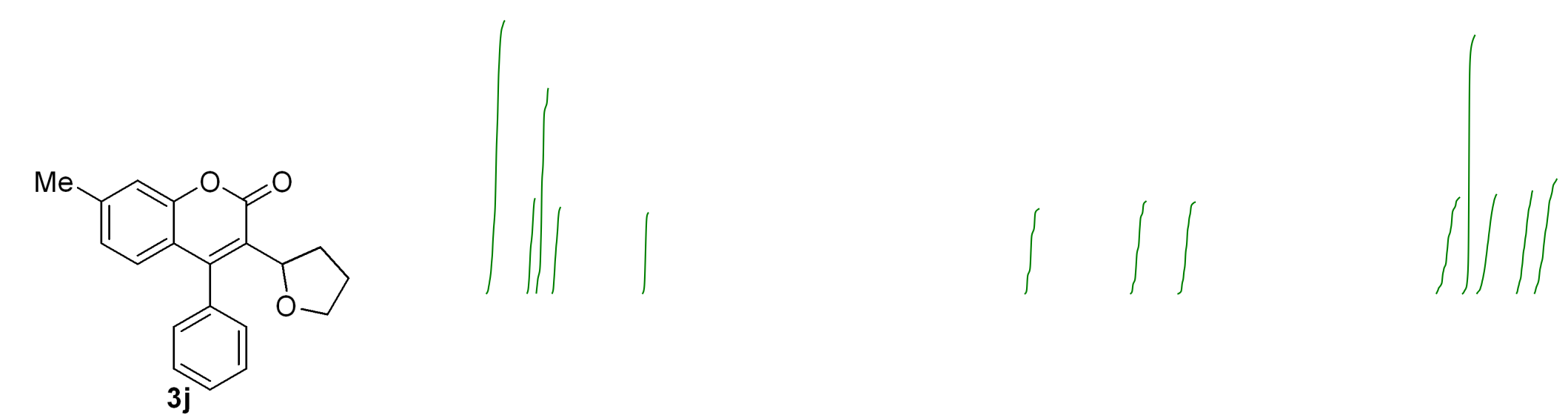

${ }^{1} \mathrm{H}$ NMR $\left(400 \mathrm{MHz}, \mathrm{CDCl}_{3}\right)$

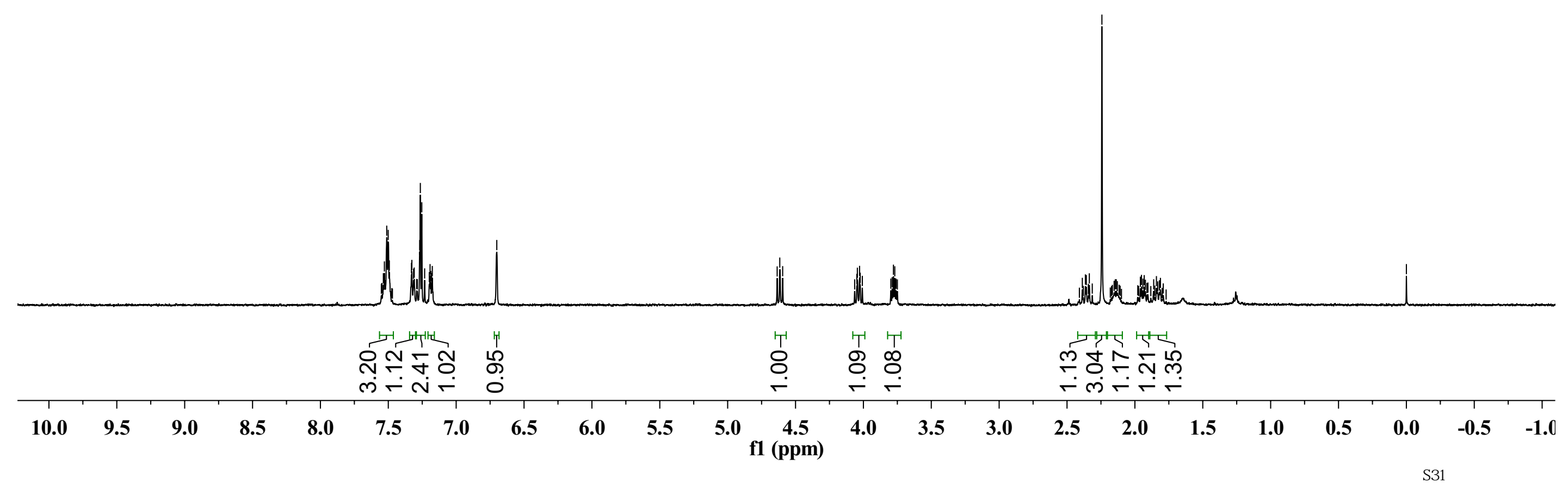




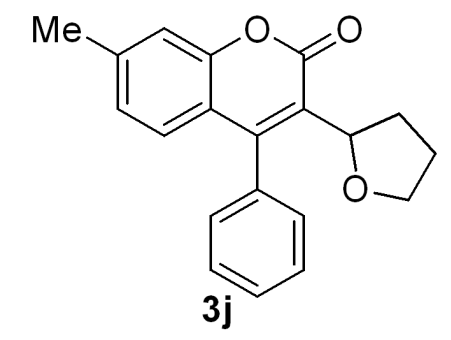

${ }^{13} \mathrm{C}$ NMR (100 MHz, $\left.\mathrm{CDCl}_{3}\right)$

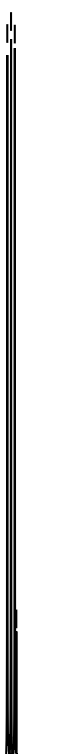



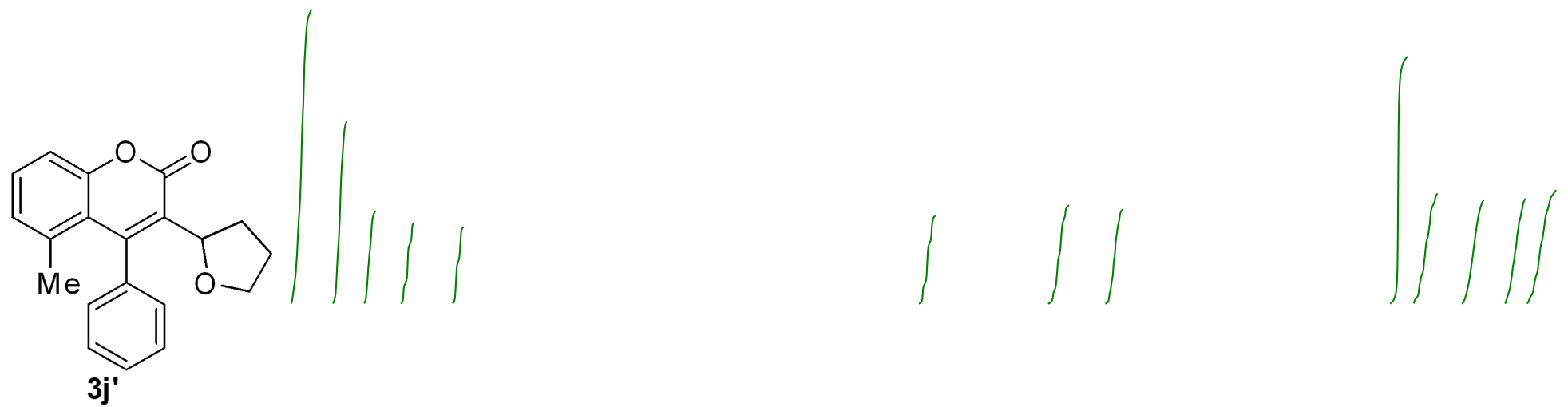

${ }^{1} \mathrm{H}$ NMR $\left(400 \mathrm{MHz} \mathrm{CDCl}_{3}\right)$

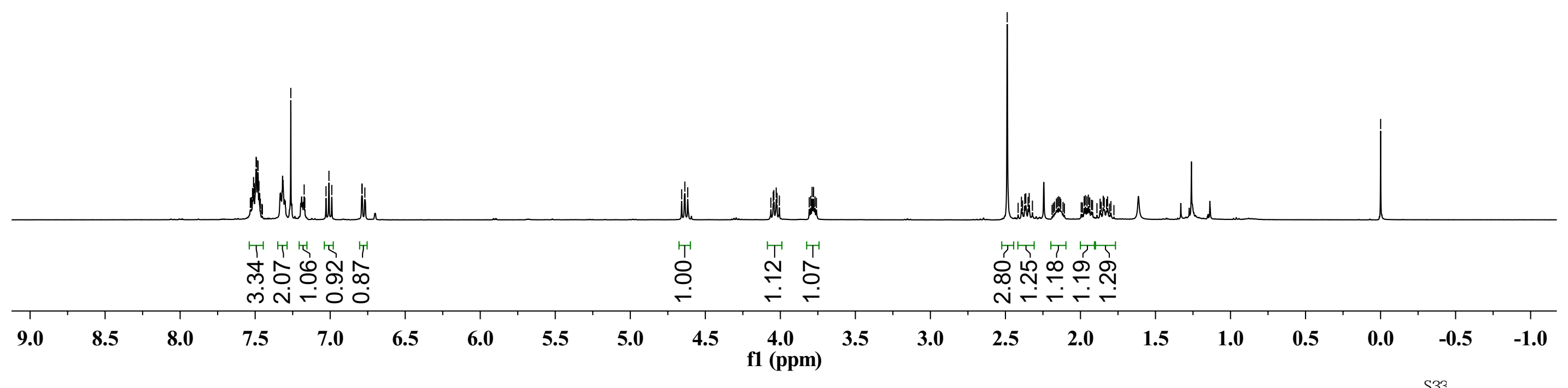




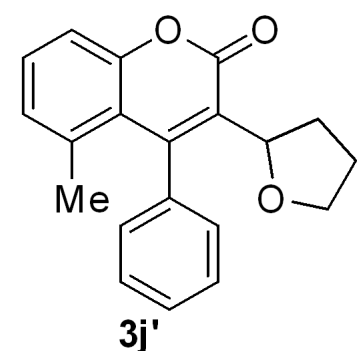

${ }^{13} \mathrm{C}$ NMR $\left(100 \mathrm{MHz}, \mathrm{CDCl}_{3}\right)$

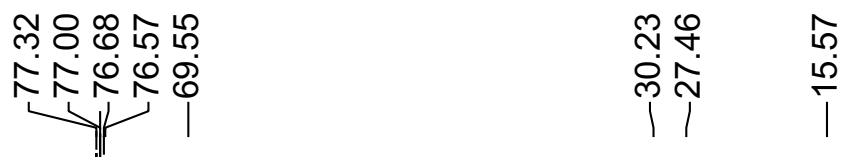

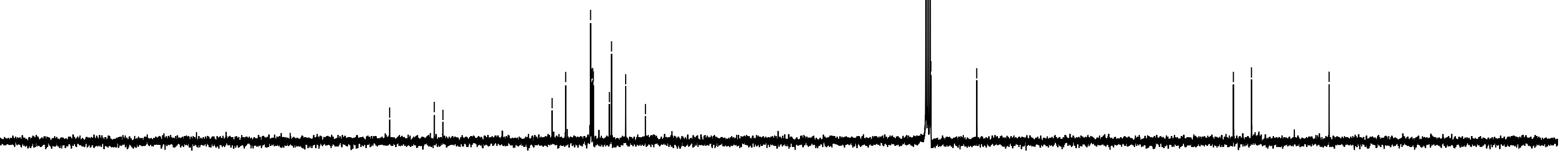

$\begin{array}{lllllllllllllllllllllllllllll}210 & 200 & 190 & 180 & 170 & 160 & 150 & 140 & 130 & 120 & 110 & \begin{array}{c}100 \\ \text { f1 (ppm) }\end{array} & 90 & 80 & 70 & 60 & 50 & 40 & 30 & 20 & 10 & 0 & -10 & \end{array}$



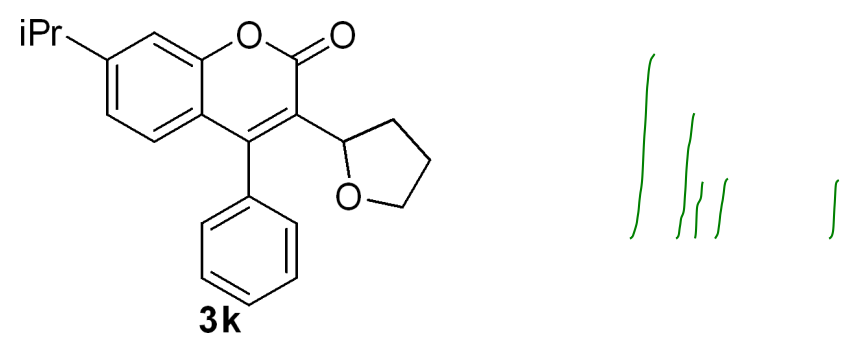

${ }^{1} \mathrm{H}$ NMR $\left(400 \mathrm{MHz}, \mathrm{CDCl}_{3}\right)$

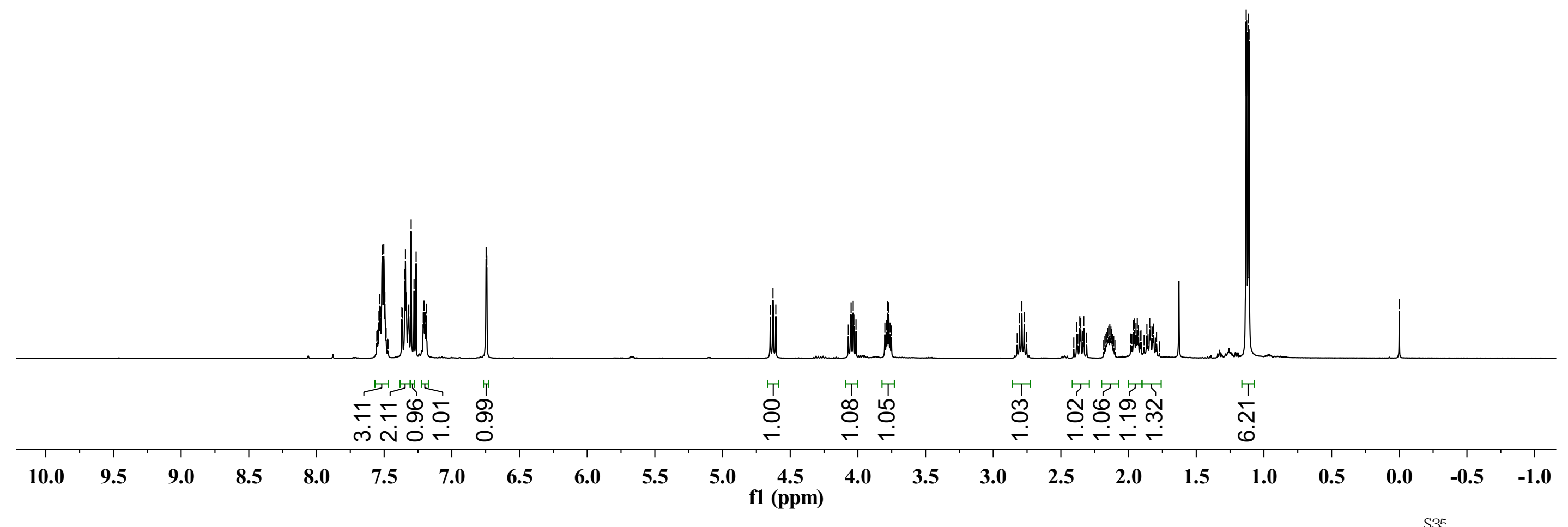




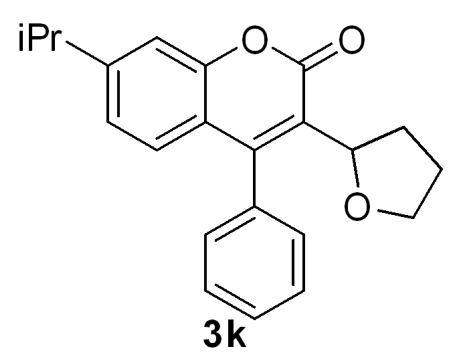

${ }^{13} \mathrm{C}$ NMR (100 MHz, $\mathrm{CDCl}_{3}$ )

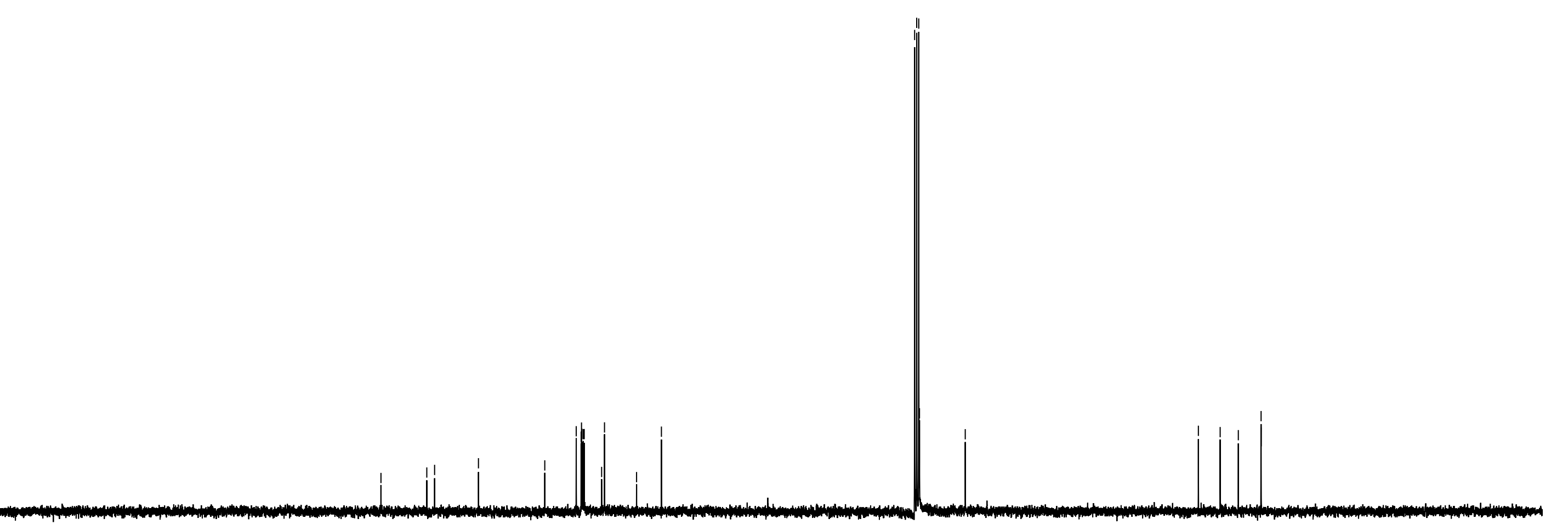

\begin{tabular}{|c|c|c|c|c|c|c|c|c|c|c|c|c|c|c|c|c|c|c|c|c|c|c|}
\hline 210 & 200 & 190 & 180 & 170 & 160 & 150 & 140 & 130 & 120 & 110 & $\begin{array}{c}100 \\
\text { f1 (ppm) }\end{array}$ & 90 & 80 & 70 & 60 & 50 & 40 & 30 & 20 & 10 & 0 & -10 \\
\hline
\end{tabular}


<smiles>Cc1cc2oc(=O)c(C3CCCO3)c(-c3ccccc3)c2cc1C</smiles>

${ }^{1} \mathrm{H} \mathrm{NMR}\left(400 \mathrm{MHz}, \mathrm{CDCl}_{3}\right)$

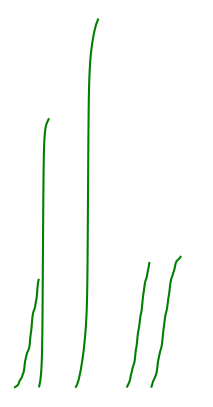

$\left.\right|^{\mid}$

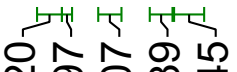

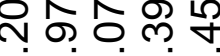
- N

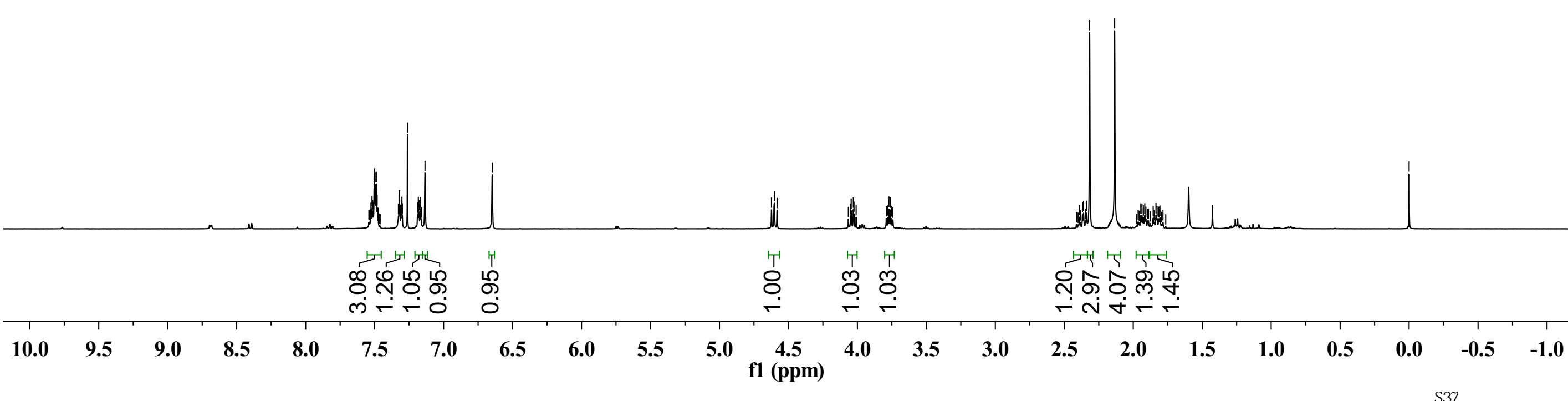




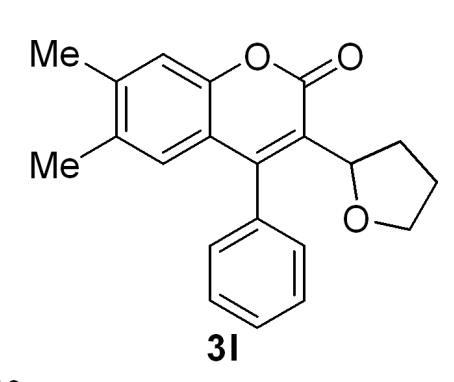

${ }^{13} \mathrm{C}$ NMR (100 MHz, $\left.\mathrm{CDCl}_{3}\right)$

กิ

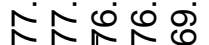

$\begin{array}{lllllllllllllllllllllllll}210 & 200 & 190 & 180 & 170 & 160 & 150 & 140 & 130 & 120 & 110 & 100 & 90 & 80 & 70 & 60 & 50 & 40 & 30 & 20 & 10 & 0 & -10 & & \end{array}$



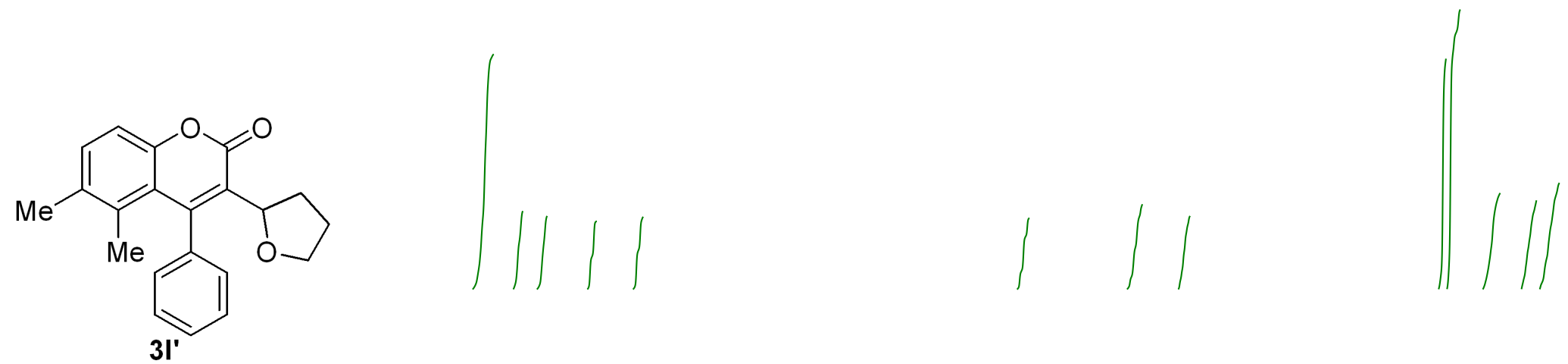

${ }^{1} \mathrm{H}$ NMR $\left(400 \mathrm{MHz}, \mathrm{CDCl}_{3}\right)$

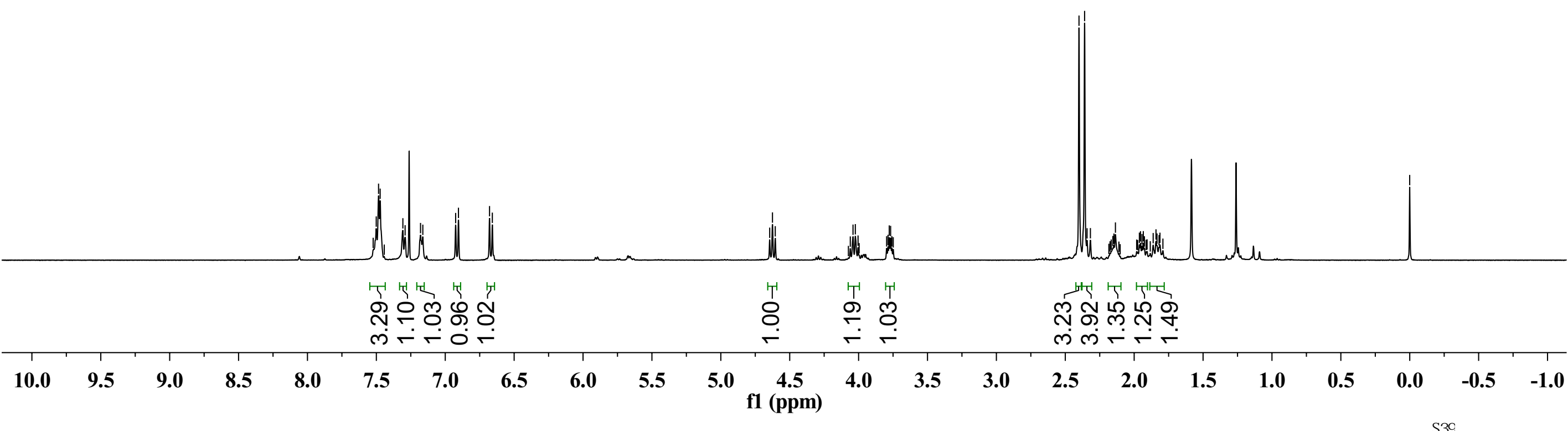




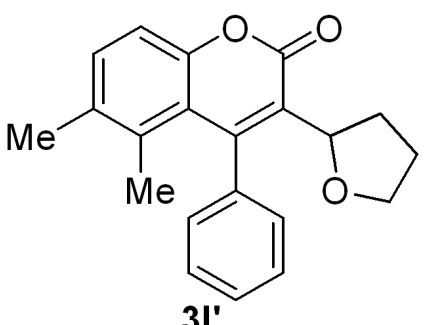

${ }^{13} \mathrm{C}$ NMR (100 MHz, $\mathrm{CDCl}_{3}$ )

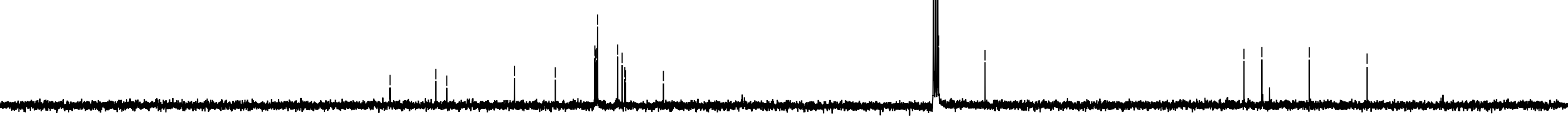



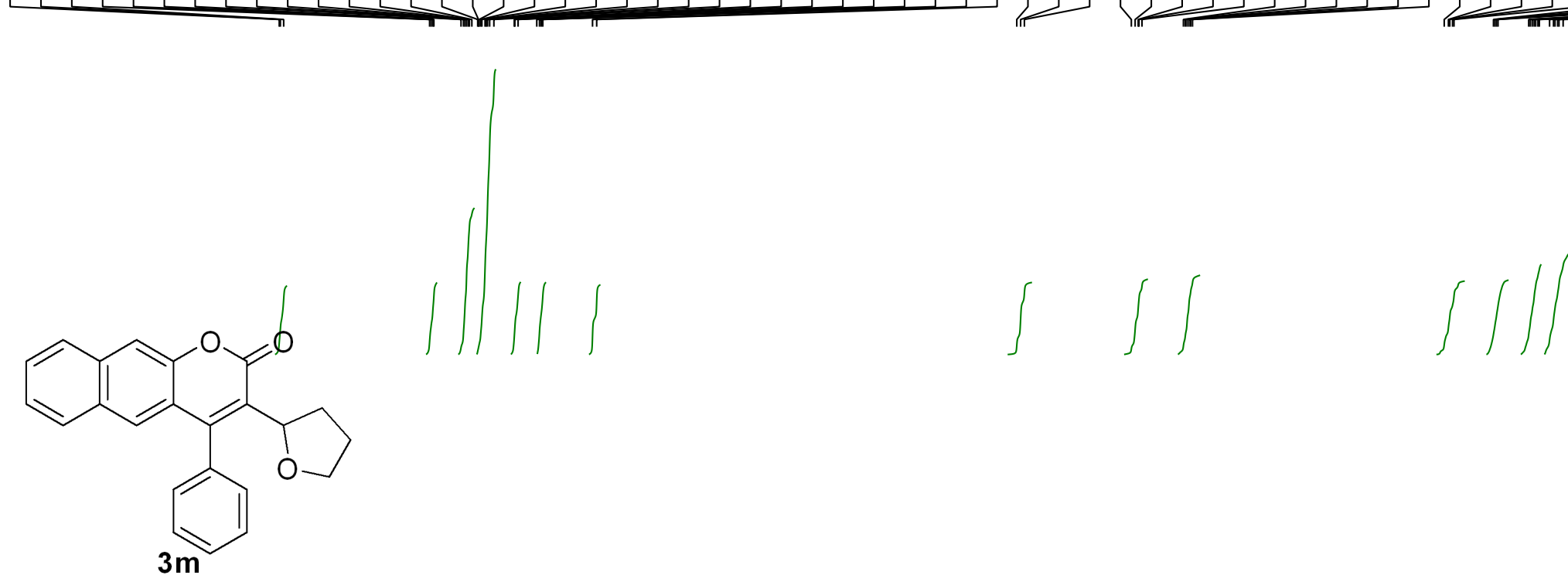

${ }^{1} \mathrm{H}$ NMR $\left(400 \mathrm{MHz}, \mathrm{CDCl}_{3}\right)$

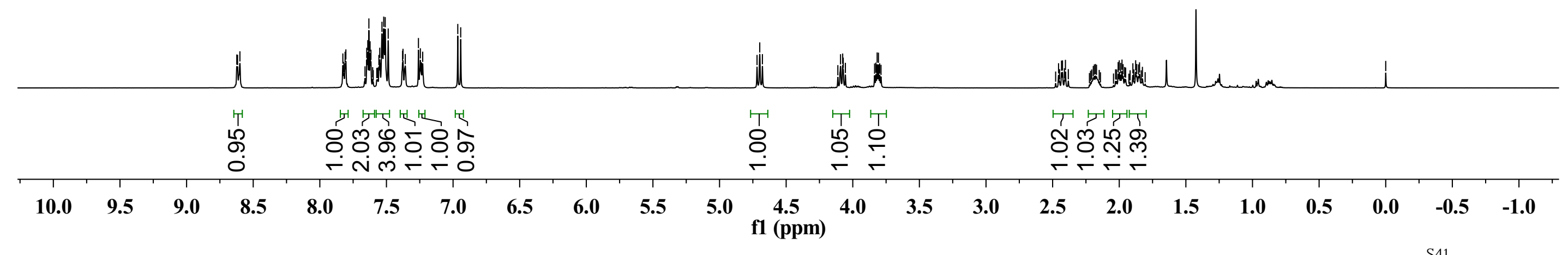




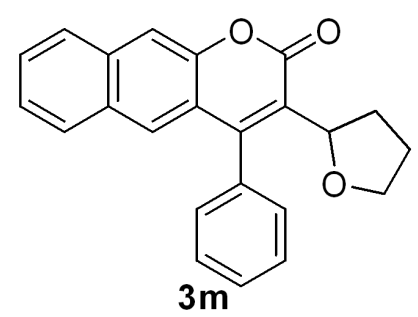

${ }^{13} \mathrm{C}$ NMR (100 MHz, $\left.\mathrm{CDCl}_{3}\right)$

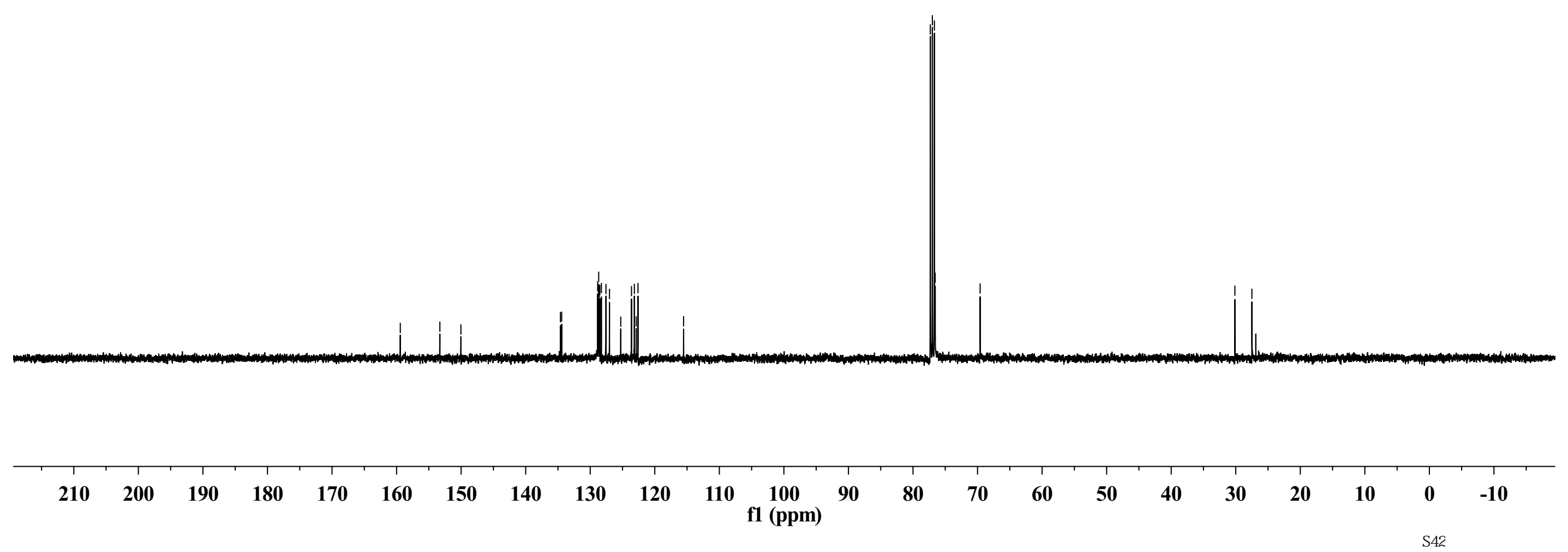




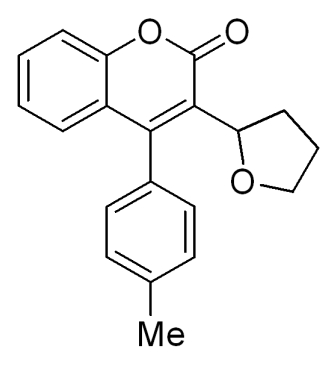

30
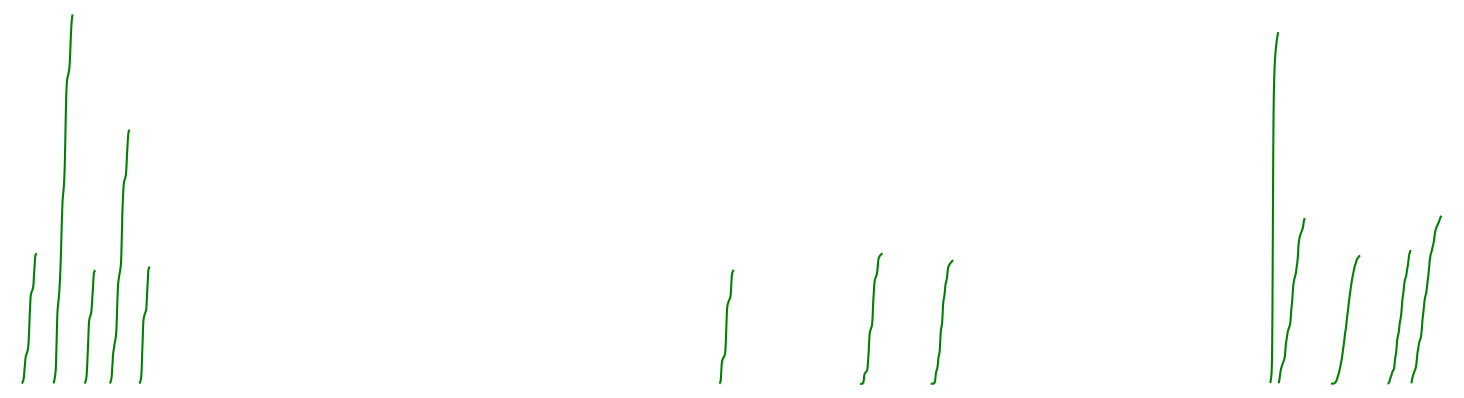

${ }^{1} \mathrm{H}$ NMR (400 $\left.\mathrm{MHz} \mathrm{CDCl}_{3}\right)$

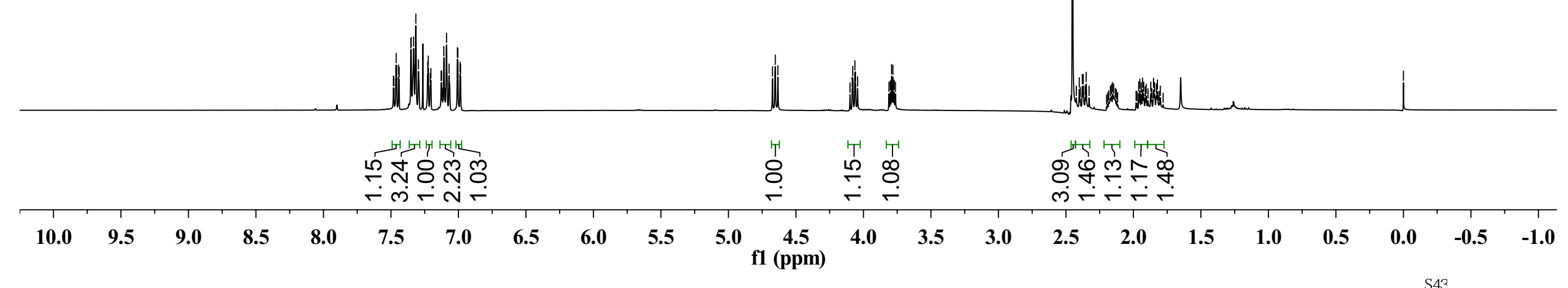




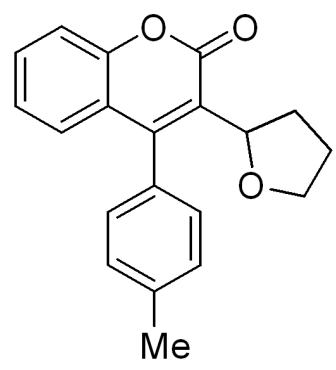

30

${ }^{13} \mathrm{C}$ NMR (100 MHz, CDCl $\left.{ }_{3}\right)$

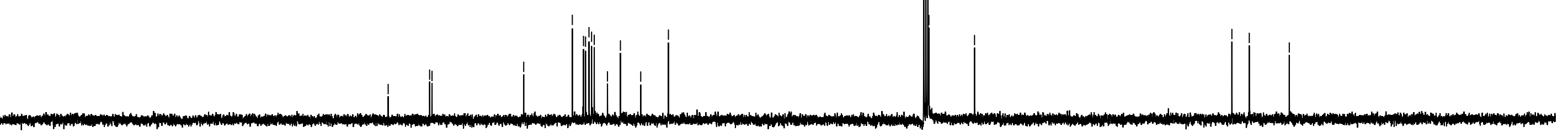



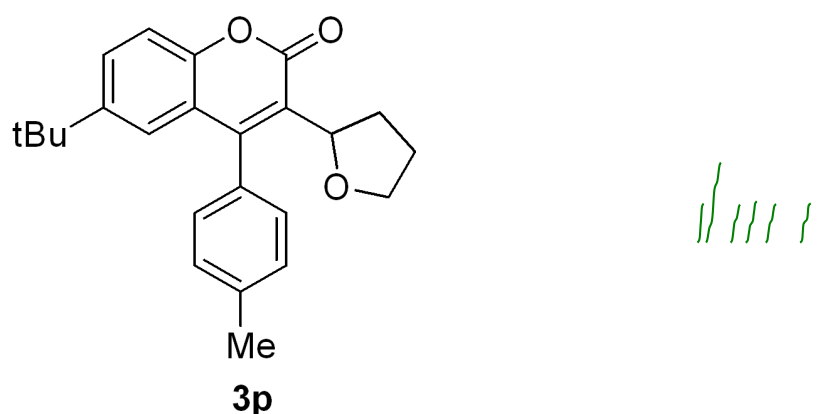

${ }^{1} \mathrm{H}$ NMR (400 MHz, $\left.\mathrm{CDCl}_{3}\right)$
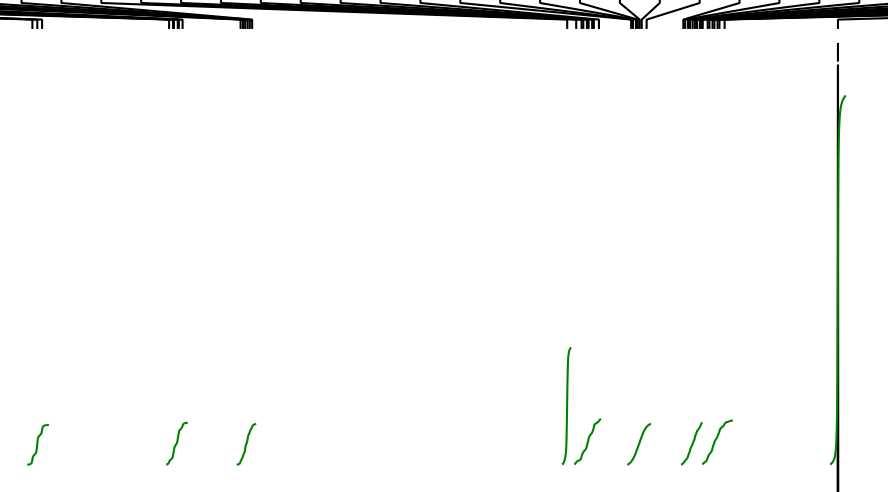


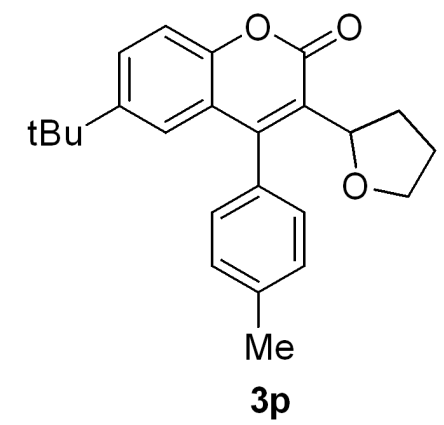

${ }^{13} \mathrm{C}$ NMR (100 MHz, $\left.\mathrm{CDCl}_{3}\right)$

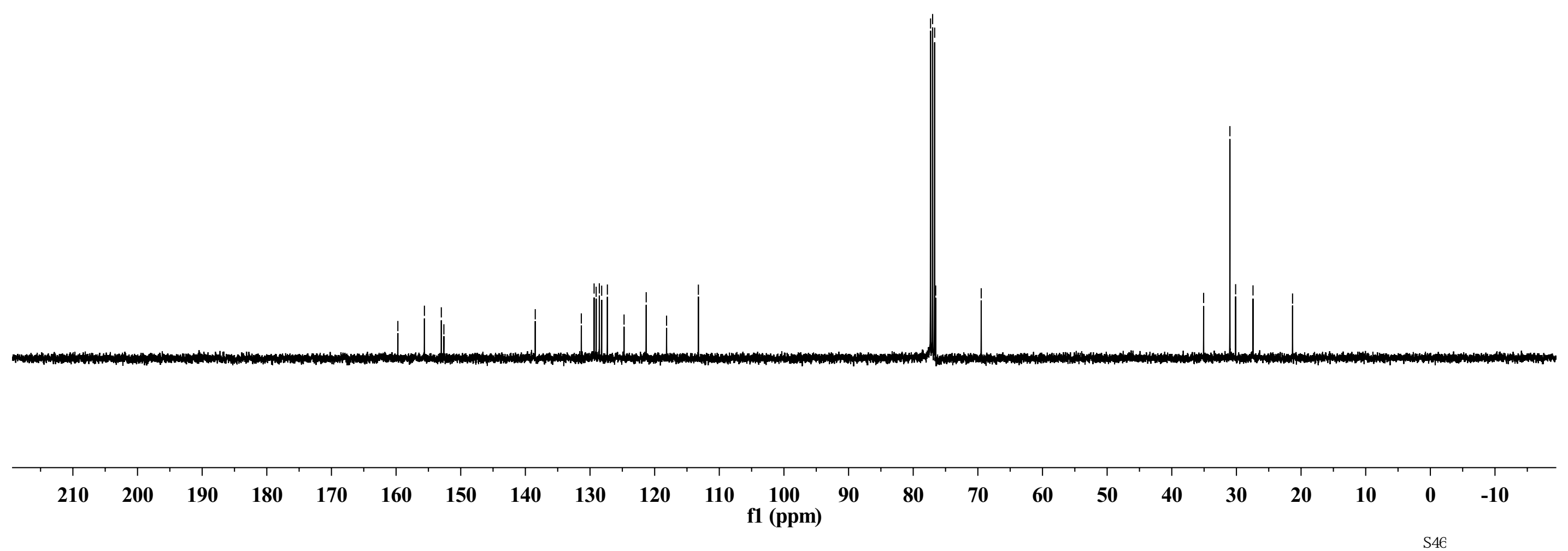


<smiles>CC(C)(C)c1ccc2oc(=O)c(C3CCCO3)c(-c3ccc(Cl)cc3)c2c1</smiles>

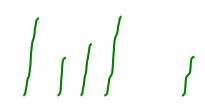

$3 q$

${ }^{1} \mathrm{H}$ NMR $\left(400 \mathrm{MHz}, \mathrm{CDCl}_{3}\right)$

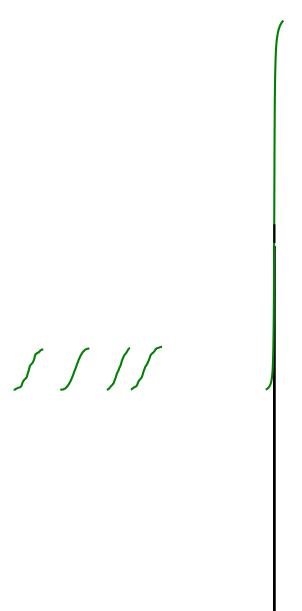

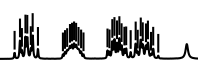

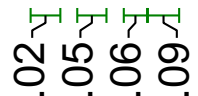

\section{$\stackrel{\mathfrak{n}}{n}$} के - O - N f1 (ppm) 


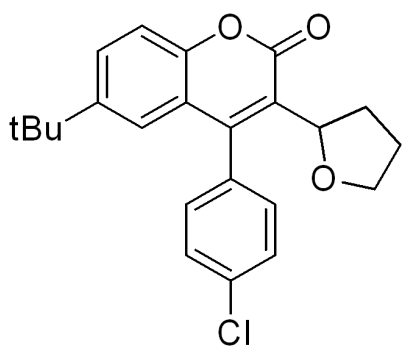

$3 q$

${ }^{13} \mathrm{C} \mathrm{NMR}\left(100 \mathrm{MHz}, \mathrm{CDCl}_{3}\right)$

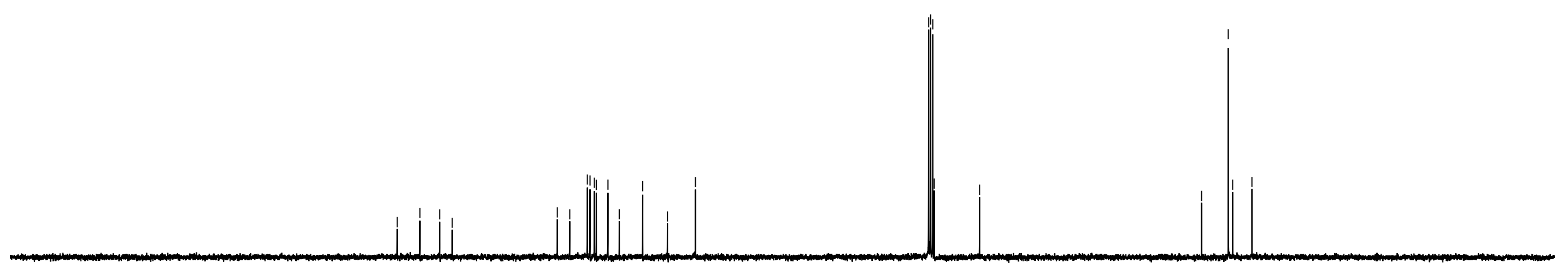

70



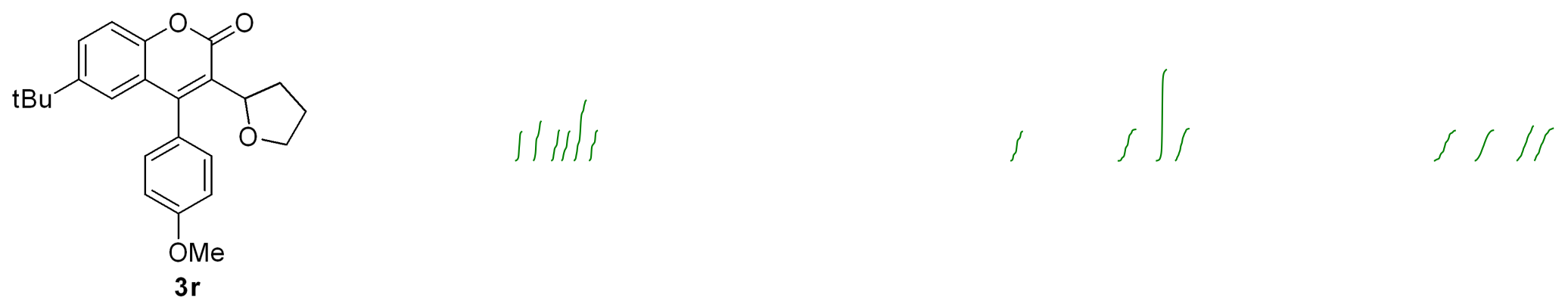

${ }^{1} \mathrm{H}$ NMR $\left(400 \mathrm{MHz}, \mathrm{CDCl}_{3}\right)$

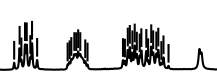

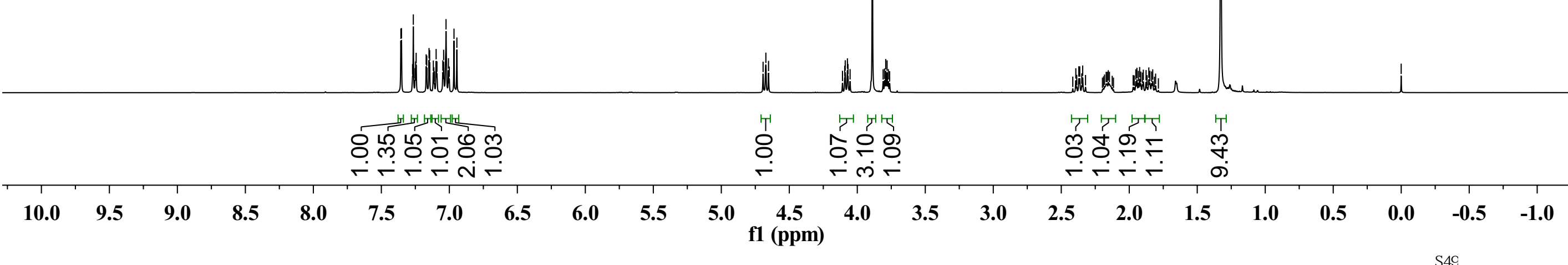




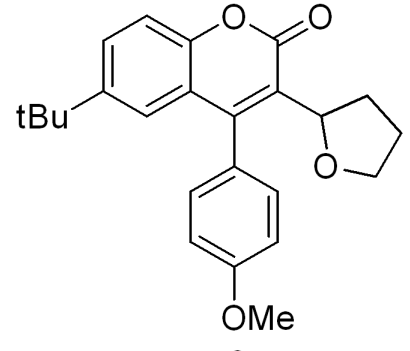

$3 r$

${ }^{13} \mathrm{C}$ NMR (100 MHz, $\left.\mathrm{CDCl}_{3}\right)$

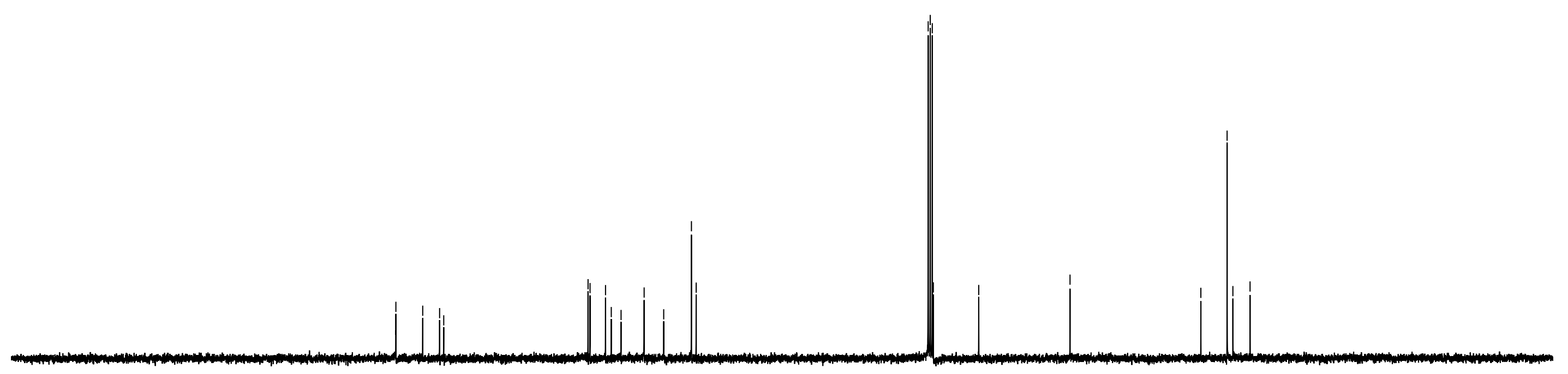


<smiles>CC(C)(C)c1ccc2oc(=O)c(C3CCCO3)c(-c3ccsc3)c2c1</smiles>

$3 \mathrm{~s}$

${ }^{1} \mathrm{H}$ NMR (400 MHz, $\left.\mathrm{CDCl}_{3}\right)$

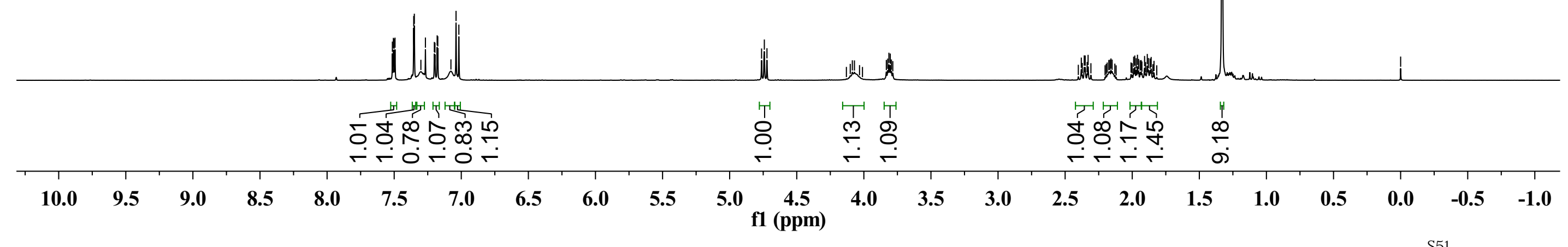



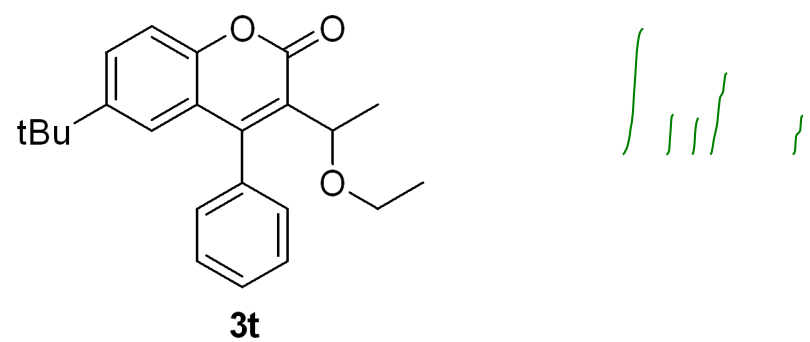

${ }^{1} \mathrm{H}$ NMR (400 MHz, $\left.\mathrm{CDCl}_{3}\right)$
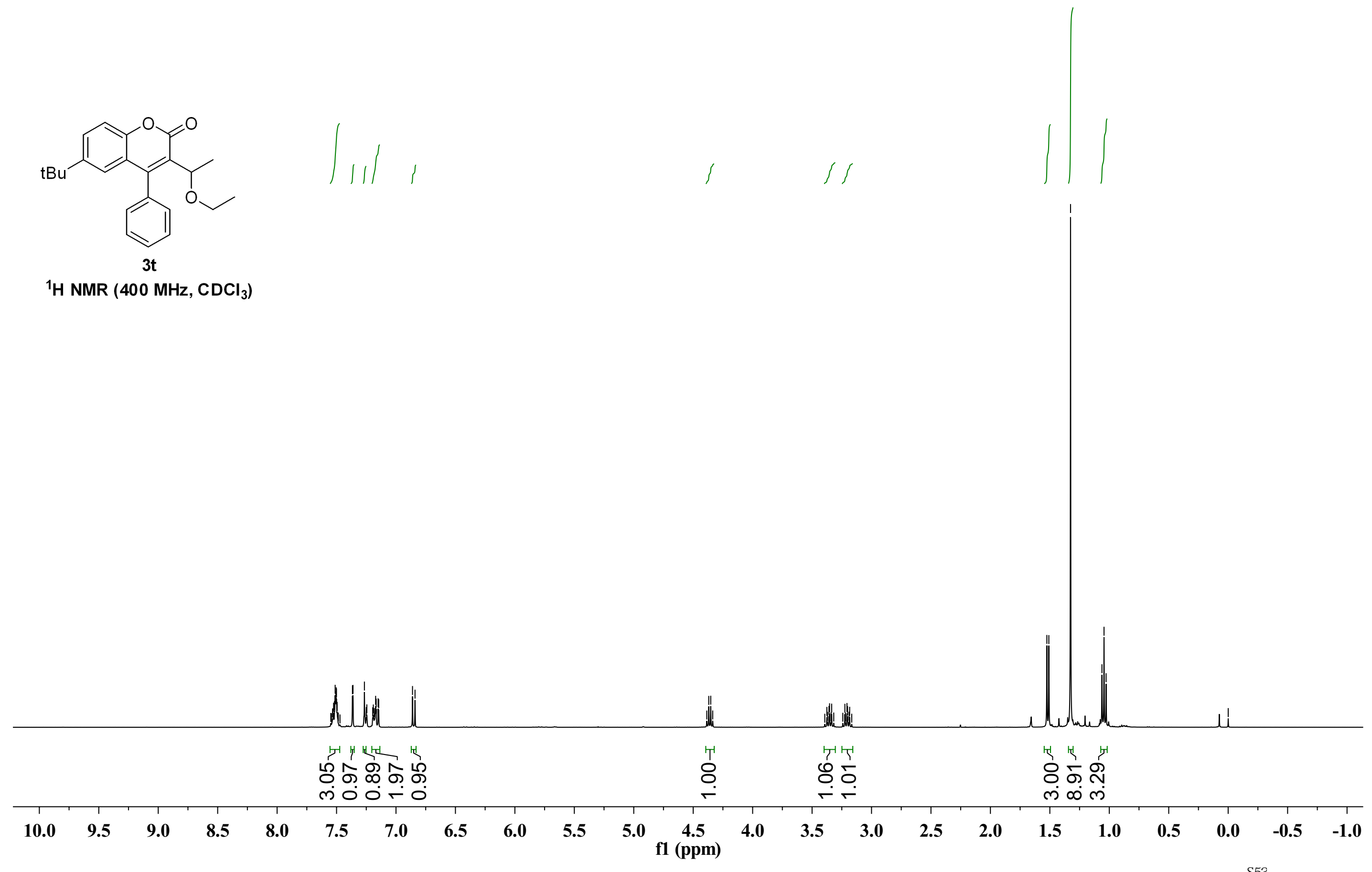


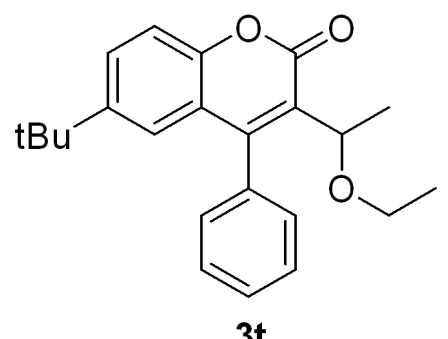

${ }^{13} \mathrm{C}$ NMR (100 MHz, $\left.\mathrm{CDCl}_{3}\right)$

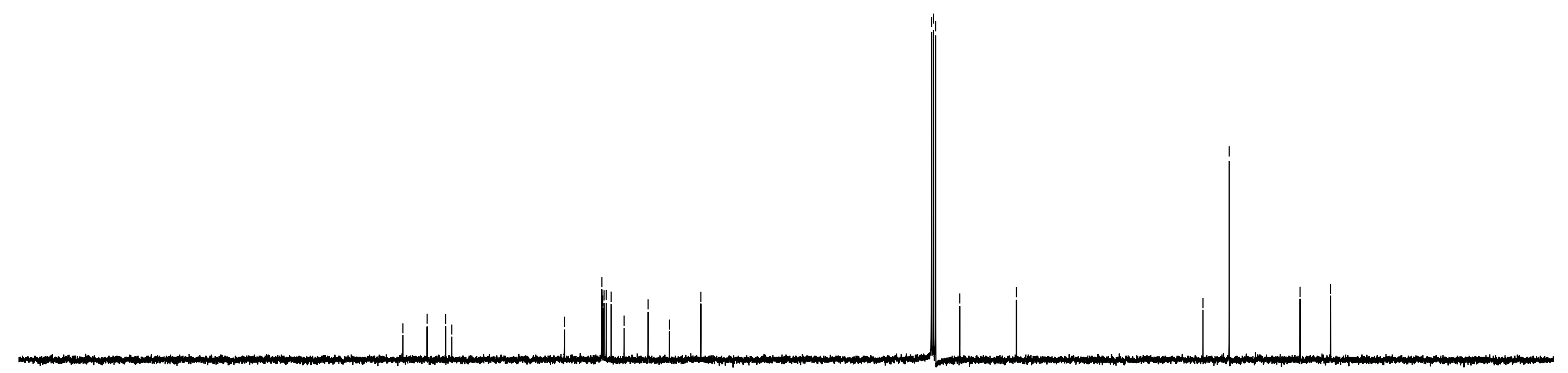



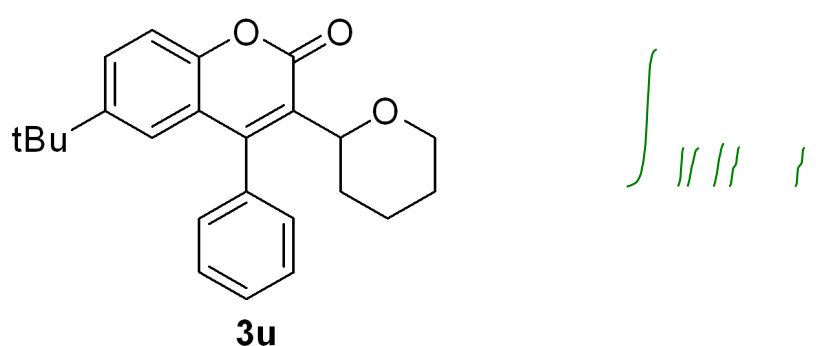

${ }^{1} \mathrm{H}$ NMR (400 MHz, $\left.\mathrm{CDCl}_{3}\right)$

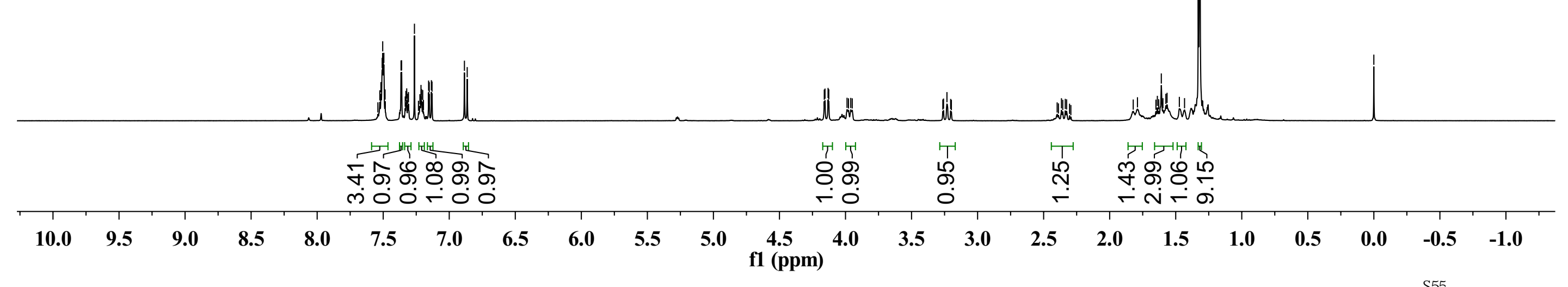




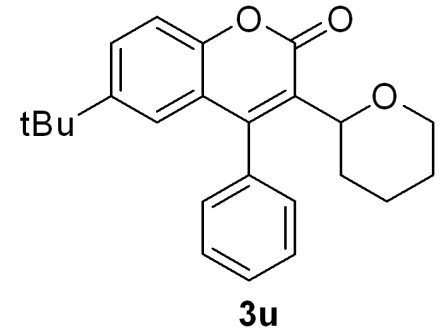

${ }^{13} \mathrm{C}$ NMR (100 MHz, $\left.\mathrm{CDCl}_{3}\right)$

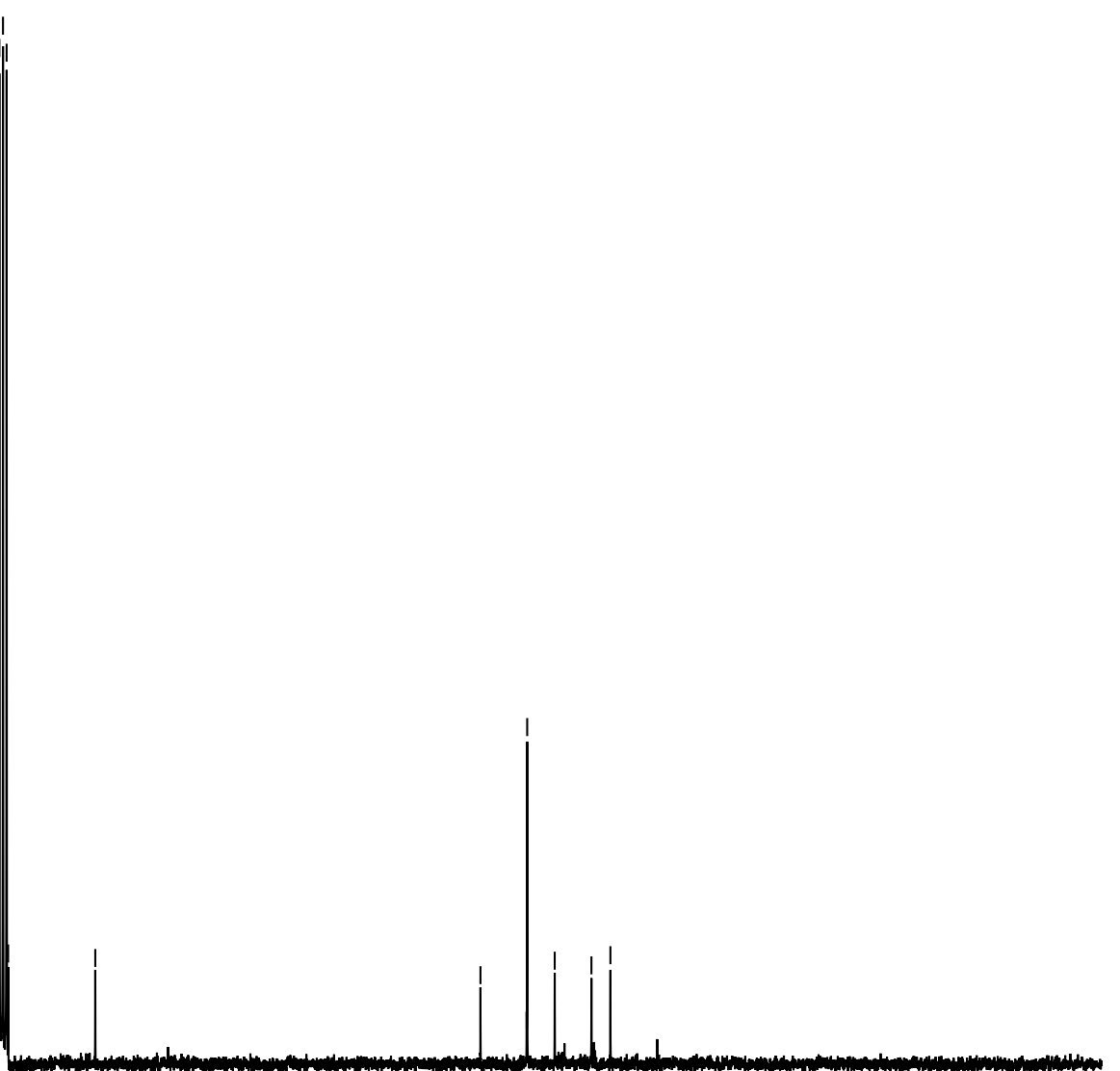

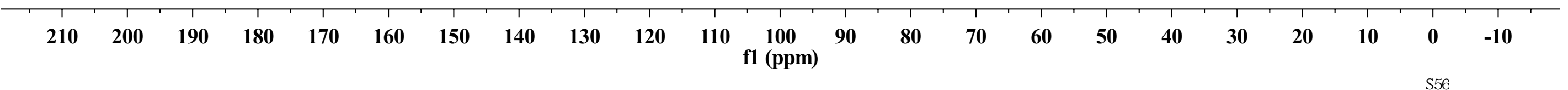


<smiles>CC(C)(C)c1ccc2oc(=O)c(C3COCCO3)c(-c3ccccc3)c2c1</smiles>

$3 \mathrm{v}$

${ }^{1} \mathrm{H}$ NMR $\left(400 \mathrm{MHz}, \mathrm{CDCl}_{3}\right)$
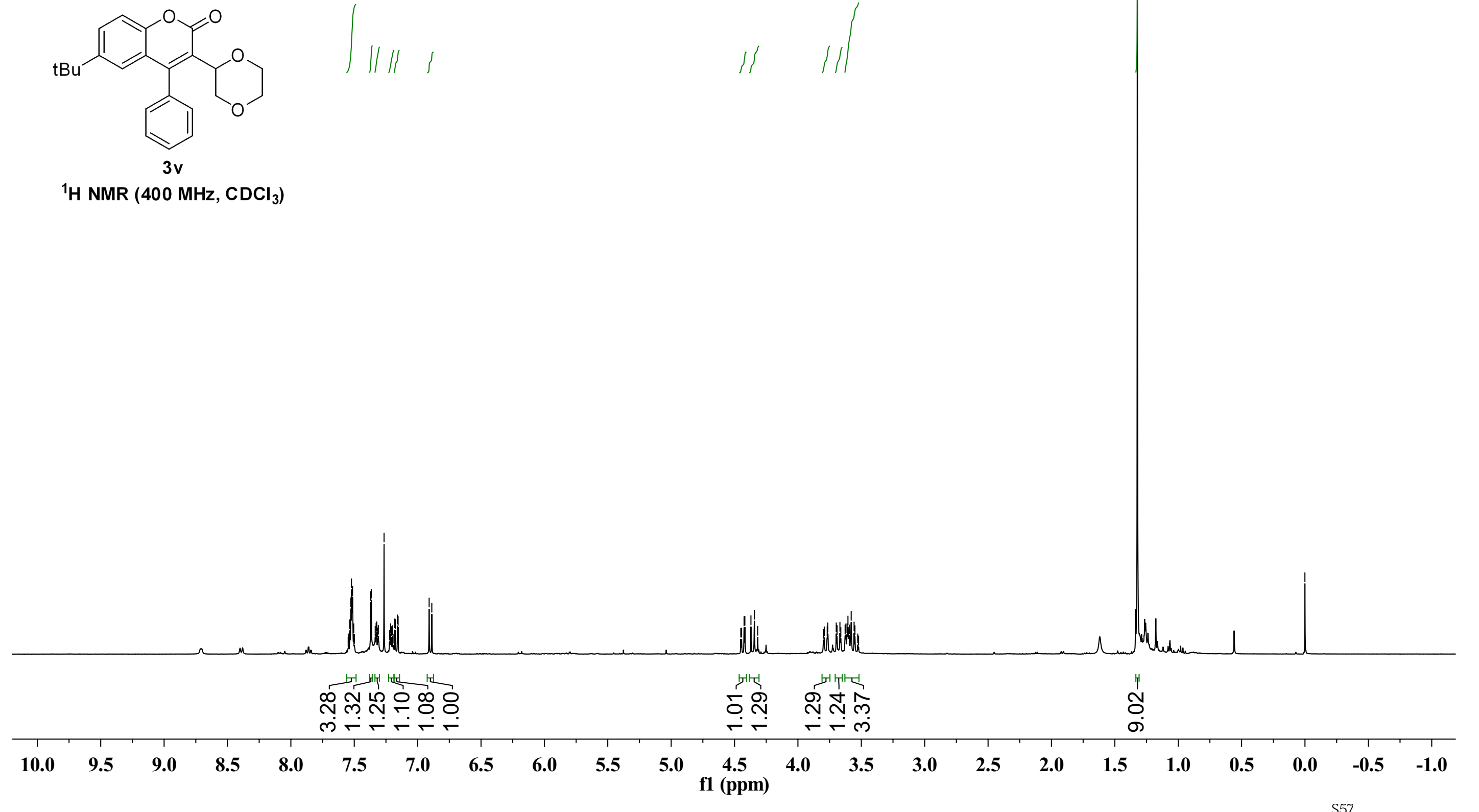


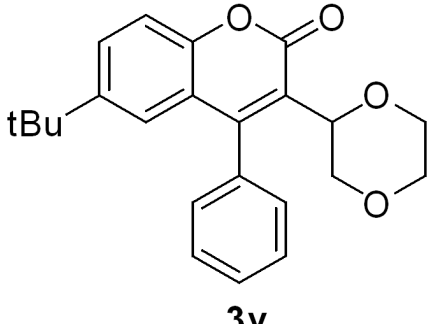

$3 v$

${ }^{13} \mathrm{C}$ NMR $\left(100 \mathrm{MHz}, \mathrm{CDCl}_{3}\right)$ 
<smiles>CC(C)(C)c1ccc2oc(=O)c(C3OCCO3)c(-c3ccccc3)c2c1</smiles>

$3 w$

${ }^{1} \mathrm{H}$ NMR $\left(400 \mathrm{MHz}, \mathrm{CDCl}_{3}\right)$
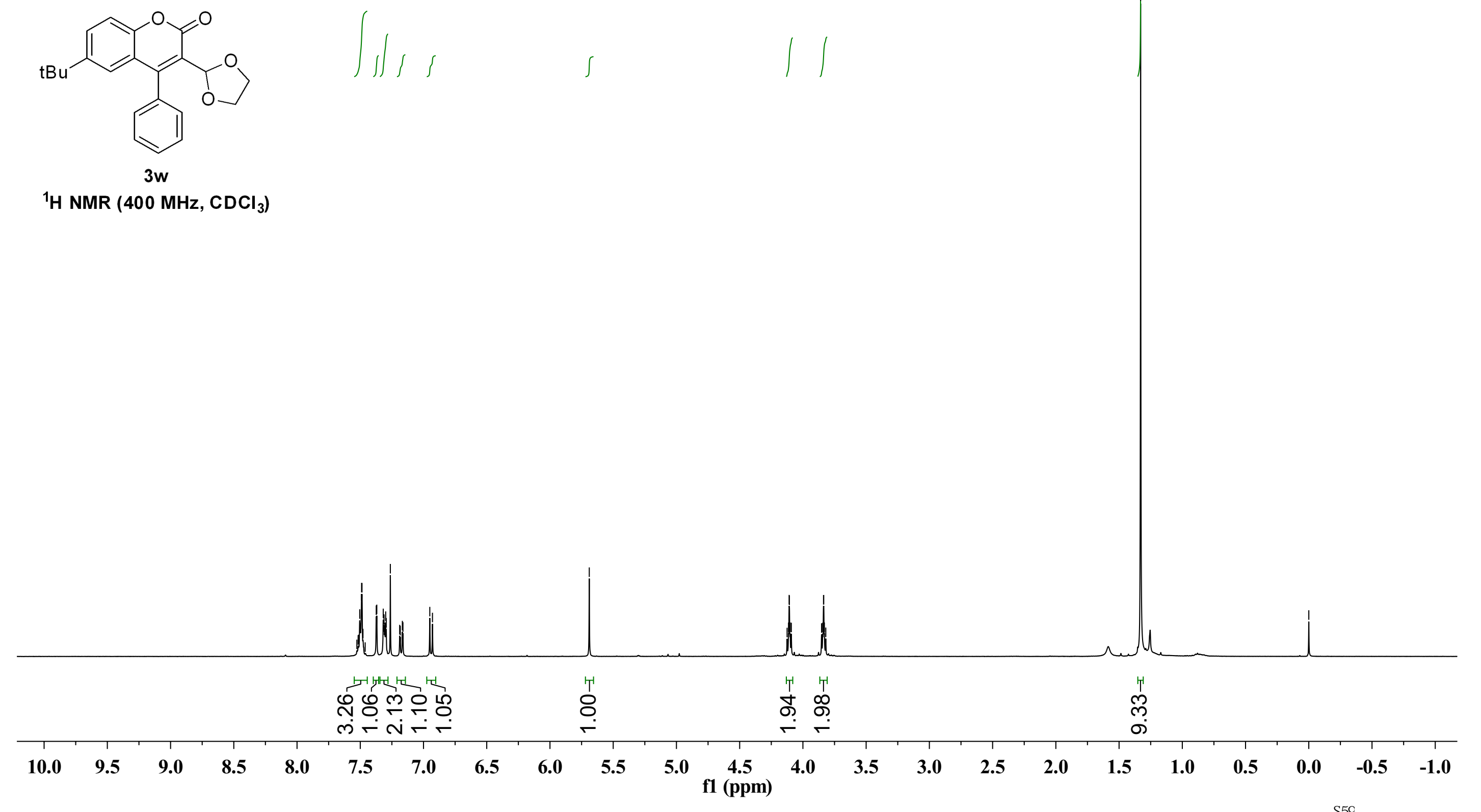
लि ठे

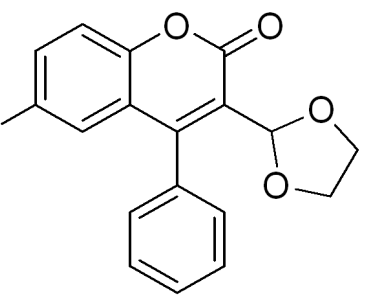

$3 w$

${ }^{13} \mathrm{C}$ NMR (100 MHz, $\mathrm{CDCl}_{3}$ ) 
<smiles>COCC(OC)c1c(-c2ccccc2)c2cc(C(C)(C)C)ccc2oc1=O</smiles>

$3 x$<smiles>COCCOCc1c(-c2cccc(C)c2)c2cc(C(C)(C)C)ccc2oc1=O</smiles>

\& $\hat{\infty}$

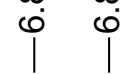

$\stackrel{m}{\stackrel{0}{i}} \underset{i}{i}$
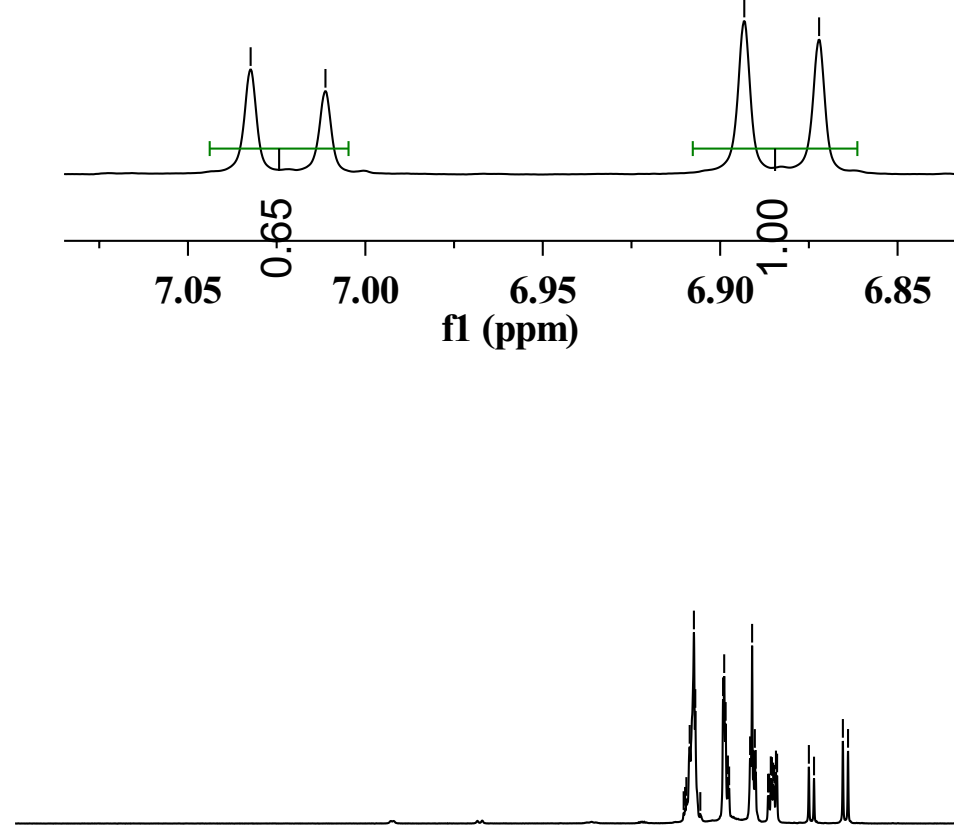

융ํㅇㅇㅛ

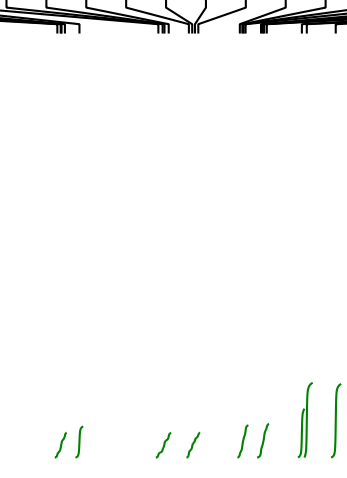

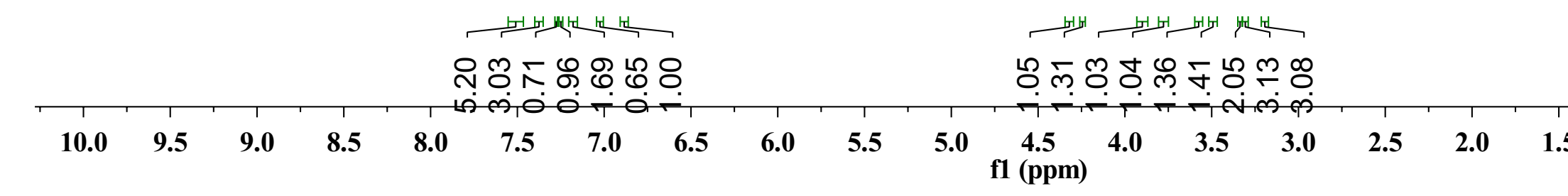



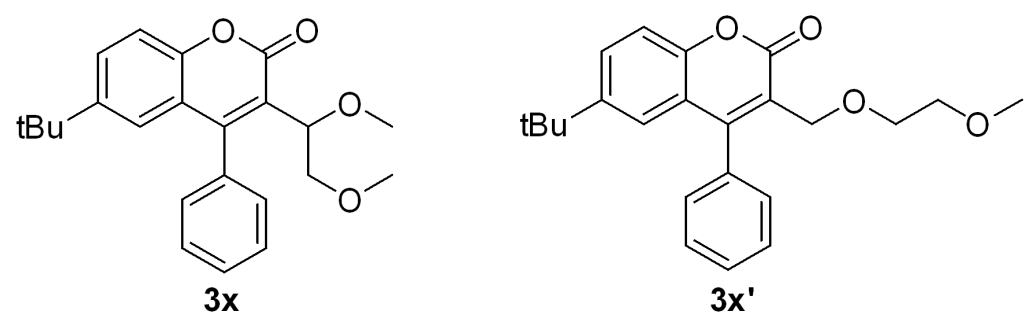

${ }^{13} \mathrm{C} \mathrm{NMR}\left(100 \mathrm{MHz}, \mathrm{CDCl}_{3}\right)$

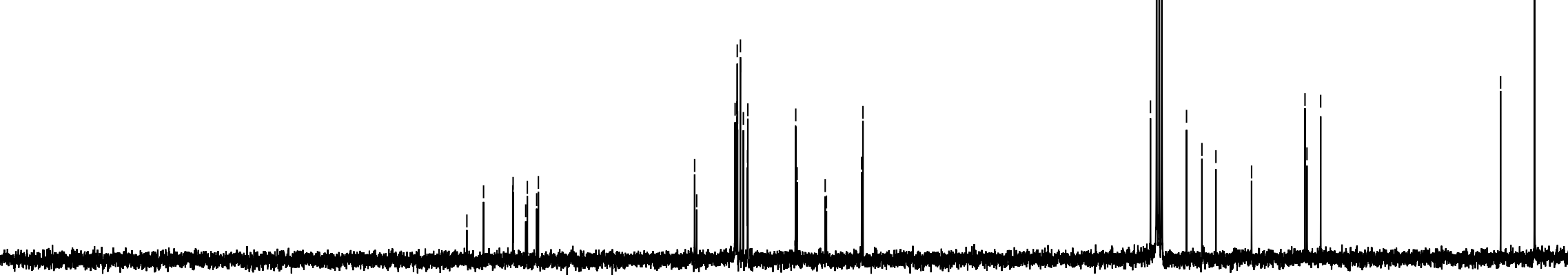

$\begin{array}{llllllllllllllllllllllllll}210 & 200 & 190 & 180 & 170 & 160 & 150 & 140 & 130 & 120 & 110 & 100 & 90 & 80 & 70 & 60 & 50 & 40 & 30 & 20 & 10 & 0 & -10 & \text { f1 (ppm) }\end{array}$




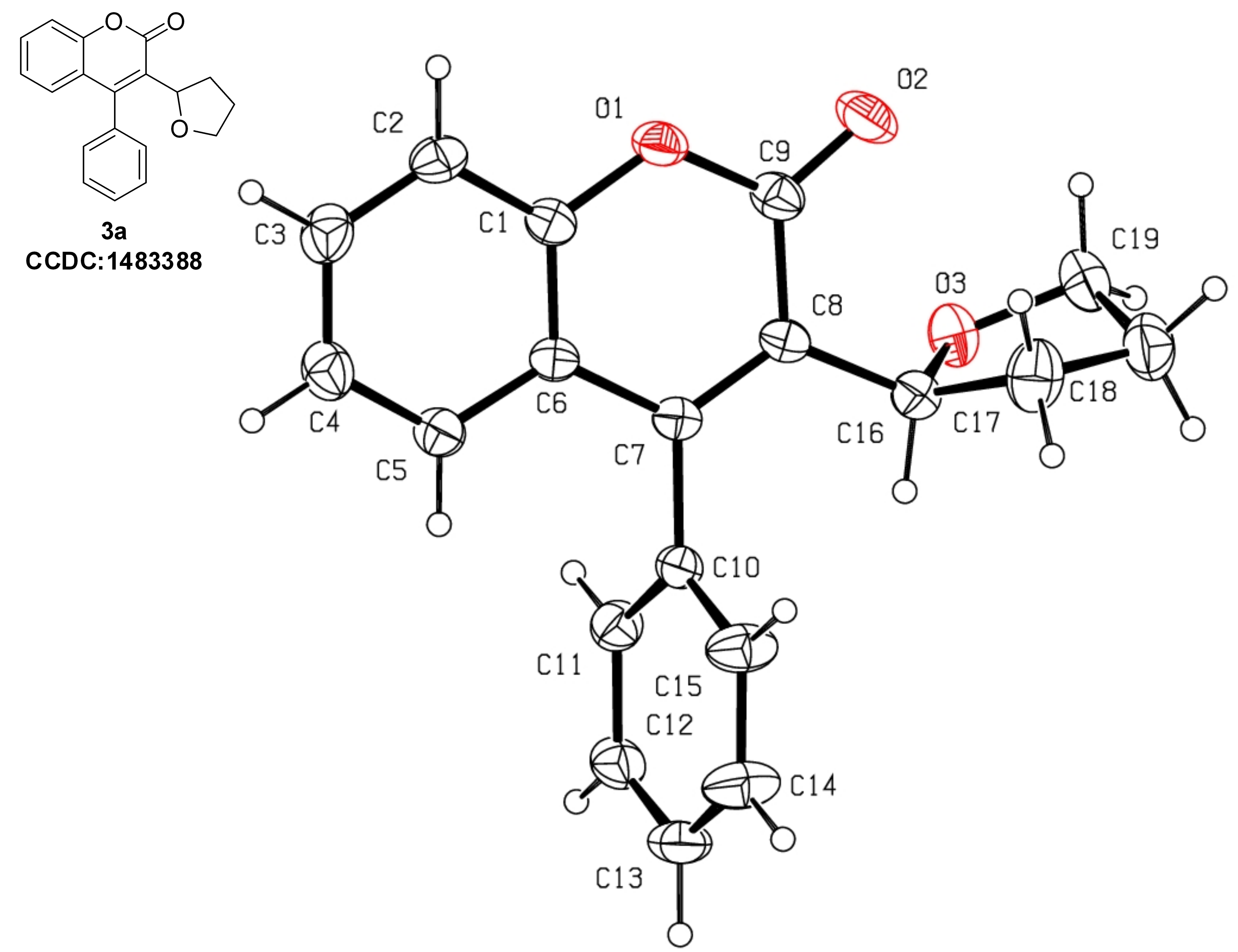

